\title{
Recent geomagnetic variations and the force balance in Earth's core
}

\author{
Julien Aubert ${ }^{\oplus}$ \\ Université de Paris, Institut de physique du globe de Paris, CNRS, F-75005 Paris, France.E-mail: aubert@ipgp.fr
}

Accepted 2020 January 6. Received 2020 January 6; in original form 2019 October 1

\begin{abstract}
SUMMAR Y
The nature of the force balance that governs the geodynamo is debated. Recent theoretical analyses and numerical simulations support a quasigeotrophic (QG), magneto-ArchimedesCoriolis (MAC) balance in Earth's core, where the Coriolis and pressure forces equilibrate at leading order in amplitude, and where the buoyancy, Lorentz and ageostrophic Coriolis forces equilibrate at the next order. In contrast, earlier theoretical expectations have favoured a magnetostrophic regime where the Lorentz force would reach leading order at the system scale. The dominant driver (buoyant or magnetic) for the general circulation in Earth's core is equally debated. In this study, these questions are explored in the light of the high-quality geomagnetic data recently acquired by satellites and at magnetic ground observatories. The analysis involves inverse geodynamo modelling, a method that uses multivariate statistics extracted from a numerical geodynamo model to infer the state of Earth's core from a geomagnetic field model interpretation of the main field and secular variation data. To test the QG-MAC dynamic hypothesis against the data, the framework is extended in order to explicitly prescribe this force balance into the inverse problem solved at the core surface. The resulting inverse solutions achieve a quantitatively adequate fit to the data while ensuring deviations from the QG-MAC balance (which amount to an inertial driving of the flow) lower than each of the leading forces. The general circulation imaged within the core over the past two decades confirms the existence of a planetary-scale, eccentric, axially columnar gyre that comprises an intense, equatorially symmetric jet at high latitudes in the Pacific hemisphere. The dominant driver of this circulation is shown to be of buoyant nature, through a thermal wind balance with a longitudinally hemispheric buoyancy anomaly distribution. Geomagnetic forecasts initiated with the inverted core states are systematically more accurate against the true interannual geomagnetic field evolution when enforcing the QG-MAC constraint. This force balance is therefore consistent with the geomagnetic data at the large scales of Earth's core that can be imaged by the method.
\end{abstract}

Key words: Dynamo: theories and simulations; Rapid time variations; Satellite magnetics; Inverse theory.

\section{INTRODUCTION}

In the past two decades, satellite geomagnetism has complemented ground observatory measurements to provide a dramatically enhanced monitoring of Earth's internally generated magnetic field. Together with improved mathematical modelling, this wealth of data has given access to reliable determinations of the core main magnetic field and its time derivatives (known as the geomagnetic secular variation and acceleration) with temporal resolution down to about a year on the largest length scales (Finlay et al. 2016b; Lesur et al. 2017). In combination with earlier modelling efforts targeted towards the historical timescales (Wardinski \& Lesur 2012; Gillet et al. 2013; Sabaka et al. 2015), the observational progress has strongly motivated recent numerical modelling efforts towards simulations that better approach the conditions of Earth's core and better render the richness of its dynamics.

As an outcome of these efforts, direct numerical simulation of the geodynamo now provides a self-consistent interpretation of geomagnetic variations over a broad range of timescales through successive force balances organized in a hierarchy of amplitudes. At decadal timescales and longer, it has been suggested that geomagnetic variations are realistically rendered even in numerical simulations operating at moderate values of the control parameters (Christensen et al. 2012; Aubert 2018). This is so because the force balance governing the slow convective evolution of the system is in fact remarkably invariant across the parameter space (Schwaiger et al. 2019) and as Earth's core conditions are approached (Aubert et al. 2017; Aubert 2019). At leading order in amplitude, the details 
of this so-called QG-MAC balance involve a geostrophic equilibrium between the Coriolis and pressure forces. The difference between the two does however not vanish, such that the equlibrium is in fact quasi-geostrophic (QG) and a small part of the Coriolis force remains ageostrophic. About an order of magnitude below the leading-order forces (the first order), this ageostrophic Coriolis force is in balance with the buoyancy and Lorentz forces (the MAC balance). Capturing the faster, interannual geomagnetic variations in the simulations however requires simulation with parameters significantly closer to Earth's core conditions (Schaeffer et al. 2017; Aubert 2018). The key here is to enlarge the gap between the first order MAC forces and the inertial and viscous force which come at the second order in amplitude. When this is achieved, magnetoinertial Alfvén hydromagnetic waves (including torsional waves) can emerge in the simulations at the appropriate timescale (which in Earth's core is on the order of a few years, Gillet et al. 2010) and with weak enough damping. Such waves generally cause weak magnetic variation signals (Aubert 2018) except during sporadic events where they can focus on approach of the core-mantle boundary and generate pulses of magnetic acceleration that lead to geomagnetic jerks (Aubert \& Finlay 2019).

Another area of research where the links between geomagnetic variations and the force balance in Earth's core are evident is the inverse problem where the magnetic induction equation is used to infer the fluid flow inside Earth's core that accounts for these variations. Various hypotheses on the geodynamo force balance have frequently been critical to alleviate the inherent underdetermination of this problem. Many inversions for flow at the core surface (e.g. Jackson 1997; Hulot et al. 2002; Pais et al. 2004; Holme \& Olsen 2006) use for instance the tangentially geostrophic constraint (Le Mouël 1984; Le Mouël et al. 1985). This constraint can be thought of as the toroidal component of the QG-MAC balance, with the magnetic force considered subdominant immediately beneath the core-mantle boundary (see Section 2.3). More recently, the quasigeostrophic approximation (again naturally encompassed by the QG-MAC theoretical description) has been repeatedly invoked to solve for flow at the core surface by using its equatorial symmetry properties and to infer its axially columnar structure throughout the core (Pais \& Jault 2008; Gillet et al. 2009; Amit \& Pais 2013; Gillet et al. 2015; Kloss \& Finlay 2019). Another avenue of research has focused on using statistics from numerical dynamos in order to constrain the inverse problem at the core surface (Fournier et al. 2011; Barrois et al. 2017; Gillet et al. 2019) as well as throughout the core (Aubert 2013, 2014, 2015). Together with the buoyancy field that can also be statistically inferred that way, the resulting flows respect a thermal wind balance between the buoyancy and ageostrophic Coriolis forces. This is expected because this balance is also generally observed at large scales in the numerical dynamos used as prior models (Aubert 2015; Aubert et al. 2017), and has a significant signature in the linear correlations between observed and hidden quantities that are used for the inversion. Here again, the thermal wind balance can be seen as the poloidal projection of the QG-MAC balance, again with magnetic forces neglected.

Among recent results of inverse core flow modelling, it is particularly noteworthy that the general circulation throughout the core has become rather consensual. Numerous studies indeed highlight a planetary-scale, eccentric and axially columnar gyre that accounts for the core surface geomagnetic westward drift beneath the Atlantic hemisphere and features a strong, high-latitude localized jet beneath the Pacific (Pais \& Jault 2008; Gillet et al. 2013, 2015, 2019; Aubert 2013, 2014, 2015; Pais et al. 2015; Barrois et al. 2017; Livermore et al. 2017; Bärenzung et al. 2018). The dominant driver of this circulation is however still debated between the two end-members of a thermal wind origin (e.g. Aubert et al. 2013; Aubert 2015) or a magnetic wind origin (e.g. Livermore et al. 2017), the latter representing a situation where it is the magnetic force, rather than buoyancy, that equilibrates the pressure and Coriolis forces at the large, planetary scale. This debate in fact reflects the more general theoretical question of whether quasi-geostrophy is indeed enforced at the system scale (as theoretically advocated by a number of authors, e.g. Davidson 2013; Calkins et al. 2015; Aurnou \& King 2017; Calkins 2018) or whether this scale is controlled by magnetostrophy, that is a leading-order equilibrium between magnetic, Coriolis and pressure forces, as historically conjectured (e.g. Hollerbach 1996; Dormy 2016). The recent numerical simulations of Schwaiger et al. (2019) suggest that although magnetostrophy can occur in the system, the scale at which it occurs is significantly below the system scale. Furthermore, the possibility of magnetic winds is generally hampered in the simulations by the effects of Lenz' law which align magnetic field lines along axial vorticity structures (Aubert 2019). The question of whether Earth's core is in a QG-MAC or system-scale magnetostrophic force balance nevertheless remains central to the geodynamo theory (Aurnou \& King 2017), and to the geophysical relevance of numerical dynamo simulations in general.

The purpose of this study is to pursue a data-driven approach to this question and use the recent high-quality geomagnetic data collected by satellites and at ground observatories in an attempt to provide further insight into the nature of force balance in Earth's core. To this end, it is necessary to infer the hidden state variables of the core from the data in a dynamically consistent way. In its previous implementations, inverse geodynamo modelling (Aubert 2013, 2014, 2015) has provided inferences of these variables that were largely of probabilistic nature (except for the determination of core surface flow from the secular variation by inversion of the magnetic induction equation). Here, the framework is further developed in order to also hypothesize the QG-MAC balance at the core surface as a prior and include the corresponding dynamical constraints in the inversion scheme. As these constraints involve the velocity field but not its time derivative, the geomagnetic acceleration is not needed to probe the dynamics, and the secular variation data remain sufficient. A quantitative evaluation of the compatibility of observed geomagnetic variations with this prior force balance hypothesis then becomes possible (with the high-accuracy recent data providing the tightest checks), which forms the first goal of this study. Our second goal is then to determine the dominant driver of flows at large scales within Earth's core. Among the tools that can be used to reach these two goals, the updated scheme can be used to perform short-term forecasts of the geomagnetic field evolution, and as a third side goal we also wish to examine the quality of these forecasts from an operational point of view. The manuscript is organized as follows: Section 2 presents the numerical dynamo and geomagnetic field models, together with the inverse geodynamo modelling scheme. Results are presented in Section 3 and discussed in Section 4.

\section{MODELS AND METHODS}

\subsection{Governing equations of the direct problem and numerical implementation}

Earth's outer core is modelled as an electrically conducting and rotating spherical fluid shell of thickness $D=r_{o}-r_{i}=2260 \mathrm{~km}$ 
between the inner core and core-mantle boundary radii $r_{i}$ and $r_{o}$, with $r_{i} / r_{o}=0.35$. In this domain, the equations of Boussinesq rotating convection, magnetic induction in the magnetohydrodynamic approximation and thermochemical density anomaly transport are solved. The unknowns are the velocity field $\mathbf{u}$, magnetic field $\mathbf{B}$, pressure field $\Pi$ and codensity (opposite of density anomaly) field $C$. In dimensional form these equations and the solenoidal conditions on $\mathbf{u}$ and $\mathbf{B}$ write:

$$
\begin{aligned}
&-2 \Omega \mathbf{e}_{z} \times \mathbf{u}-\frac{\nabla \Pi}{\rho}+g_{o} \frac{\mathbf{r}}{\rho r_{o}} C+\frac{1}{\rho \mu_{0}}(\nabla \times \mathbf{B}) \times \mathbf{B} \\
&= \frac{\mathrm{D} \mathbf{u}}{\mathrm{D} t}-v \nabla^{2} \mathbf{u}, \\
& \frac{\partial \mathbf{B}}{\partial t}=\nabla \times(\mathbf{u} \times \mathbf{B})+\eta \nabla^{2} \mathbf{B}, \\
& \frac{\partial C}{\partial t}+\mathbf{u} \cdot \nabla C=\kappa \nabla^{2} C+\chi, \\
& \nabla \cdot \mathbf{u}=0, \nabla \cdot \mathbf{B}=0 .
\end{aligned}
$$

Here $\Omega \mathbf{e}_{z}$ is the rotation vector of the planetary reference frame in which the equations are solved, $\mathbf{r}$ is the radius vector, $g_{o}$ the gravity at radius $r_{o}, \rho, \mu_{0}, \nu, \kappa$ and $\eta$ are, respectively, the fluid density, magnetic permeability, viscous, thermo-chemical and magnetic diffusivities and $\chi$ is a source term ensuring mass conservation. In eq. (1) the terms relevant to the QG-MAC force balance have been grouped in the left-hand side, such that in the models as well as at Earth's core conditions (Aubert et al. 2017; Aubert 2018), individual terms in this balance dominate the residual of their sum in the right-hand side, that comprises inertia (represented here as a total time derivative) and viscosity. As demonstrated in Aubert (2018) also, the dynamics of the system on decadal and longer timescales, as well as on shorter timescales away from jerk events, is governed by the balance between terms in the left-hand side of eq. (1), with the temporal dependence being prescribed by the time derivatives present in eqs (2) and (3) rather than by inertia in eq. (1).

The specific model used here is the coupled Earth dynamo (Aubert et al. 2013), which has already served a number of times in geomagnetic inverse problems (Aubert 2014, 2015; Finlay et al. 2016a; Barrois et al. 2017). Rigid (no-slip) and stress-free mechanical boundary conditions are prescribed at the inner core (coremantle) boundary, respectively. The inner core is electrically conducting and insulating electromagnetic boundary conditions are prescribed at the core-mantle boundary. A gravitational restoring torque couples the inner core and the mantle. As a result of the electromagnetic, mechanical and gravitational couplings between the inner core, outer core and mantle, the inner core and mantle have a time-dependent axial rotation rate respectively to the outer core, and the ensemble has a constant axial angular momentum defining the planetary rotation rate $\Omega$. Fixed-flux, heterogeneous density anomaly boundary conditions are prescribed at both boundaries, with amplitudes and patterns as detailed in Aubert et al. (2013). The geophysical situation that is modelled is that of dominant bottom-driven chemical convection driven by a differentially freezing inner core. The longitudinally hemispherical pattern of the freezing rate is fastest in the Eastern Hemisphere (from longitudes $0^{\circ} \mathrm{E}$ to $180^{\circ} \mathrm{E}$ ) and peaks at $90^{\circ} \mathrm{E}$. At the core-mantle boundary, the homogeneous part of the density anomaly flux vanishes (corresponding to a situation where the heat flow is exactly adiabatic), and the density anomaly flux is locally perturbed by an heterogeneous thermal control from the mantle.

The system of eqs (1)-(4) is cast into a dimensionless form before being numerically solved (see e.g. Aubert et al. 2017). This leads to four main dimensionless parameters, the flux Rayleigh, Ekman, Prandtl and magnetic Prandtl numbers, the respective values of which for the coupled earth model are:

$$
\begin{aligned}
& R a_{F}=\frac{g_{o} F}{4 \pi \rho \Omega^{3} D^{4}}=2.7 \times 10^{-5}, \\
& E=\frac{v}{\Omega D^{2}}=3 \times 10^{-5}, \\
& P r=\frac{v}{\kappa}=1, \\
& P m=\frac{v}{\eta}=2.5 .
\end{aligned}
$$

Here $F$ is the homogeneous part of the density anomaly flux prescribed at the inner core boundary. In terms of computational requirements, the model is located within a parameter space region of moderate cost that has been extensively explored, leading in particular to a quantitative assessment of the degree of semblance between the output of numerical simulations and the geomagnetic field (Christensen et al. 2010). Against the metrics that have been introduced, the large scale output of the coupled earth model is rated highly semblant to the historical geomagnetic field, and this also true (though on a more qualitative level) of the large-scale spatial structure of its secular variation and underlying core flows (Aubert et al. 2013). Its relevance for geomagnetism has also recently been further strengthened by the formulation of a theoretical parameter space path between this model and conditions in Earth's core, along which the leading order QG-MAC force balance and the large-scale structure of the solution are approximately invariant (Aubert et al. 2017).

The properties of the parameter space path also justify the rescaling rules that have been previously defined (Fournier et al. 2011, and following studies) to cast the dimensionless model output back into dimensional values relevant to Earth's core despite the wide parameter gap existing between the two. Here we proceed in the following steps:

(i) Dimensionless lengths in the model are expressed relative to $D$ and therefore rescaled by multiplying the result with Earth's core shell gap $D=2260 \mathrm{~km}$.

(ii) We then invoke the invariance along the path of the ratio $\tau_{\mathrm{SV}} / \tau_{\eta}$ between the typical timescale for secular variation (see e.g. Christensen et al. 2012; Aubert 2018) and the magnetic diffusion time $\tau_{\eta}=D^{2} / \eta$. Dimensionless times in the model are therefore expressed relative to $\tau_{\mathrm{SV}}$ and rescaled by multiplying the result with the value $\tau_{\mathrm{SV}}=415 \mathrm{yr}$ inferred from the geomagnetic field evolution (Lhuillier et al. 2011). Using the value $\tau_{\mathrm{SV}} / \tau_{\eta}=2.96 \times$ $10^{-3}$ obtained in the coupled earth model, this is equivalent to expressing dimensionless times in the model relative to the magnetic diffusion time $\tau_{\eta}$ and then multiplying the result with the value $\tau_{\eta}=$ $140280 \mathrm{yr}$, corresponding to a magnetic diffusivity $\eta=1.15 \mathrm{~m}^{2} \mathrm{~s}^{-1}$ at the midpoint of current estimates (Aubert et al. 2017).

(iii) Dimensionless velocities in the model are expressed relative to $\eta / D$ (yielding magnetic Reynolds numbers) and then rescaled by multiplying the result with the value for Earth's core $\eta / D=$ $0.016 \mathrm{~km} \mathrm{yr}^{-1}$ obtained using the values determined above.

(iv) We invoke the invariance along the path of the ratio between the Coriolis force $-2 \Omega \mathbf{e}_{z} \times \mathbf{u}$ and buoyancy force $g_{o} \mathbf{r} C / \rho r_{o}$. The dimensionless density anomaly field is therefore expressed relative to $\rho \Omega \eta / g_{o} D$ and then rescaled by multiplying the result with the value $\rho \Omega \eta / g_{o} D=4.08 \times 10^{-8} \mathrm{~kg} \mathrm{~m}^{-3}$ obtained using $\rho=$ $11000 \mathrm{~kg} \mathrm{~m}^{-3}, \Omega=7.29 \times 10^{-5} 1 \mathrm{~s}^{-1}, g_{o}=10 \mathrm{~m} \mathrm{~s}^{-2}$ and the values determined above. 
(v) We invoke the invariance along the path of the ratio between the Coriolis force $-2 \Omega \mathbf{e}_{z} \times \mathbf{u}$ and Lorentz force $(\nabla \times \mathbf{B}) \times$ $\mathbf{B} / \rho \mu_{0}$. The dimensionless magnetic field is therefore expressed relative to the Elsasser unit $\sqrt{\rho \mu_{0} \eta \Omega}$ and then rescaled by multiplying the result with the value of $\sqrt{\rho \mu_{0} \eta \Omega}$ obtained for Earth's core. Using the values determined above and $\mu_{0}=4 \pi \times 10^{-7} \mathrm{H} \mathrm{m}^{-1}$, we should prescribe $\sqrt{\rho \mu_{0} \eta \Omega}=1.08 \mathrm{mT}$. With this unit, the r.m.s. magnetic field in the shell of the coupled earth model is $4.7 \mathrm{mT}$, compatible with the value $4 \mathrm{mT}$ inferred for Earth's core (Gillet et al. 2010). Note that prior to the formulation of the parameter space path in Aubert et al. (2017), previous studies using the same dynamo model (Aubert et al. 2013; Aubert 2014, 2015; Fournier et al. 2015; Barrois et al. 2017) proceeded along somewhat different lines of reasoning. These nevertheless arrived at a very similar result $\sqrt{\rho \mu_{0} \eta \Omega}=0.82 \mathrm{mT}$ which is also admissible given the leeway in the possible magnetic field amplitude in Earth's core. As the results presented here are largely immune to this slight difference, and to facilitate comparison with these earlier studies, we chose to retain this last value for the Elsasser unit.

Through this rescaling procedure, the dimensional outputs respect the QG-MAC balance at the same level as their dimensionless counterparts, and at the same time compare favourably with their estimates in Earth's core.

The numerical implementation involves a representation of the solenoidal vector fields in a toroidal-poloidal formalism:

$\mathbf{u}=\nabla \times(T \mathbf{r})+\nabla \times \nabla \times(P \mathbf{r})$,

$\mathbf{B}=\nabla \times(Z \mathbf{r})+\nabla \times \nabla \times(W \mathbf{r})$.

Note that the pressure $\Pi$ is not solved for as the equations that are numerically implemented involve the curl and double curl of (1). The scalar fields $P, T, W, Z, C$ representing the velocity, magnetic and density anomaly fields in the spherical coordinate system $(r, \theta$, $\varphi$ ) are expanded on a discrete radial grid $r_{j}, j=1, \ldots, N R$ with $N R$ $=184$ gridpoints $(N G=25$ points of which are in the inner core), and in a spherical harmonic representation on a fully normalized basis of complex spherical harmonic functions $Y_{l}^{m}(\theta, \varphi)$ of degree $\ell$ and order $m$, up to degree and order $\ell_{\max }=133$, according to, for example:

$P=\sum_{\ell=0}^{\ell_{\max }} \sum_{m=-\ell}^{\ell} P_{\ell}^{m}\left(r_{j}\right) Y_{\ell}^{m}(\theta, \varphi)$.

Spatial derivatives are handled with a second-order finitedifferencing scheme, and time stepping is semi-implicit with second-order accuracy. We use the spherical harmonics transform library SHTns (Schaeffer 2013) freely available at https: //bitbucket.org/nschaeff/shtns. Angular momentum conservation within the coupled core-mantle-inner core system is controlled at each time step.

\subsection{Geomagnetic field model}

For reasons of mathematical tractability as well as reasons related to the high level of expertise that is involved in separating the internal and external sources of the geomagnetic field (see a discussion in Aubert 2015), the inverse geodynamo modelling framework developed in Section 2.3 uses as input the output of a geomagnetic field model rather than direct geomagnetic satellite and ground observatory measurements. Here, we use the iteration CHAOS-6x8 of the CHAOS-6 family of field models (Finlay et al. 2016b), spanning epochs 1999.0-2019.0. This model provides a spherical harmonic global representation of the geomagnetic field that is also expanded on a spline basis in time. Spherical harmonic coefficients up to degree $\ell_{\mathrm{obs}}=13$ can be safely assumed to represent the internal magnetic field and secular variation (Hulot et al. 2015; Finlay et al. 2016b), and we therefore adopt this truncation level. The geomagnetic field model output is sampled at yearly intervals for the poloidal magnetic field coefficients at the core surface $W_{\ell}^{m}\left(r_{o}\right)$ and their first time derivative evaluated on the spline basis $\dot{W}_{\ell}^{m}\left(r_{o}\right)$, which relate to the supplied classical Gauss coefficients $g_{\ell}^{m}, h_{\ell}^{m}$ through, for example

$W_{\ell}^{m}\left(r_{o}\right)=\frac{r_{o}}{\ell}\left(\frac{a}{r_{o}}\right)^{l+2} \frac{\left(g_{\ell}^{m}-i h_{\ell}^{m}\right)}{\sqrt{2 \ell+1}}$.

Here $a=6371.2 \mathrm{~km}$ is Earth's radius, and a similar expression holds for $\dot{W}_{\ell m}\left(r_{o}\right)$. The vectors

$$
\begin{gathered}
\mathbf{d}=\left[W_{\ell}^{m}\left(r_{o}\right)\right]^{\top}, 1 \leq \ell \leq \ell_{\mathrm{obs}}, \\
\dot{\mathbf{d}}=\left[\dot{W}_{\ell}^{m}\left(r_{o}\right)\right]^{\top}, 1 \leq \ell \leq \ell_{\mathrm{obs}},
\end{gathered}
$$

where superscript ${ }^{\top}$ denotes the transpose, are the data vectors that are introduced in the inverse framework at each given epoch. Also needed are the data error covariance matrices $\mathbf{R}$ and $\dot{\mathbf{R}}$ statistically representing the deviation $\boldsymbol{\epsilon}, \dot{\boldsymbol{\epsilon}}$ of $\mathbf{d}, \dot{\mathbf{d}}$ to their true value in Earth's core:

$\mathbf{R}=E\left(\boldsymbol{\epsilon} \boldsymbol{\epsilon}^{\prime}\right), \quad \dot{\mathbf{R}}=E\left(\dot{\boldsymbol{\epsilon}} \dot{\boldsymbol{\epsilon}}^{\prime}\right)$,

where $E$ is the expected value and the prime denotes the transpose complex conjugate. We adopt diagonal data error covariance matrices with the following coefficients:

$$
R_{\ell}^{m}=\frac{r_{o}^{2}}{\ell^{2}(\ell+1)^{2}(2 \ell+1)}\left(\frac{a}{r_{o}}\right)^{2 \ell+4} \frac{\epsilon_{B}^{2}}{\ell_{\mathrm{obs}}} .
$$

Here $\epsilon_{B}$ is the error level, and a similar expression involving a level $\epsilon_{\dot{B}}$ holds for $\dot{\mathbf{R}}$. Note that eqs (12) and (16) differ slightly from the expressions given in Aubert (2015) because of the spherical harmonic basis chosen for the present analysis (as defined in Dormy 1997), particularly the fully normalized character of basis functions (while Gauss coefficients are Schmidt semi-normalized). The quantities $\epsilon_{B}, \epsilon_{\dot{B}}$ are the total amplitudes of the error on all three magnetic field components at Earth's surface, and the expression (16) evenly distributes the error among spherical harmonic degrees in a Mauersberger-Lowes spectral representation of magnetic energy at Earth's surface (the level of this flat error spectrum being marked by arrows in Figs 1a and c, with corresponding fit residuals at the core-mantle boundary being imaged in Fig. 2). For the unconstrained inversions as well as the inversions where a moderate level of the QG-MAC constraint is imposed (see Section 2.3), we adopt $\epsilon_{B}=1 \mathrm{nT}$ and $\epsilon_{\dot{B}}=2 \mathrm{nT} \mathrm{yr}^{-1}$, these values being commensurate with the typical residuals obtained when constructing CHAOS-6 (Finlay et al. 2016b). For inversions where we impose a high level of the QG-MAC constraint, we allow for more deviation from the geomagnetic field model by keeping $\epsilon_{B}=1 \mathrm{nT}$ but adopting $\epsilon_{\dot{B}}=10 \mathrm{nT} \mathrm{yr}^{-1}$.

\subsection{Ensemble-based inverse geodynamo modelling framework including the QG-MAC constraint}

The formulation of the inversion scheme follows the single-epoch ensemble Kalman filter formulation presented in Aubert (2015). We define the magnetic and hydrodynamic state vectors for the entire discretized core model 


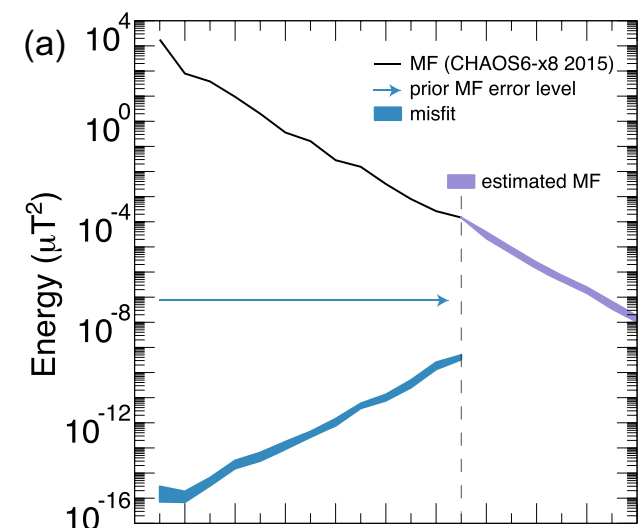

(b) analysed MF ensemble average, 2015, $\ell_{\text {asm }}=30$
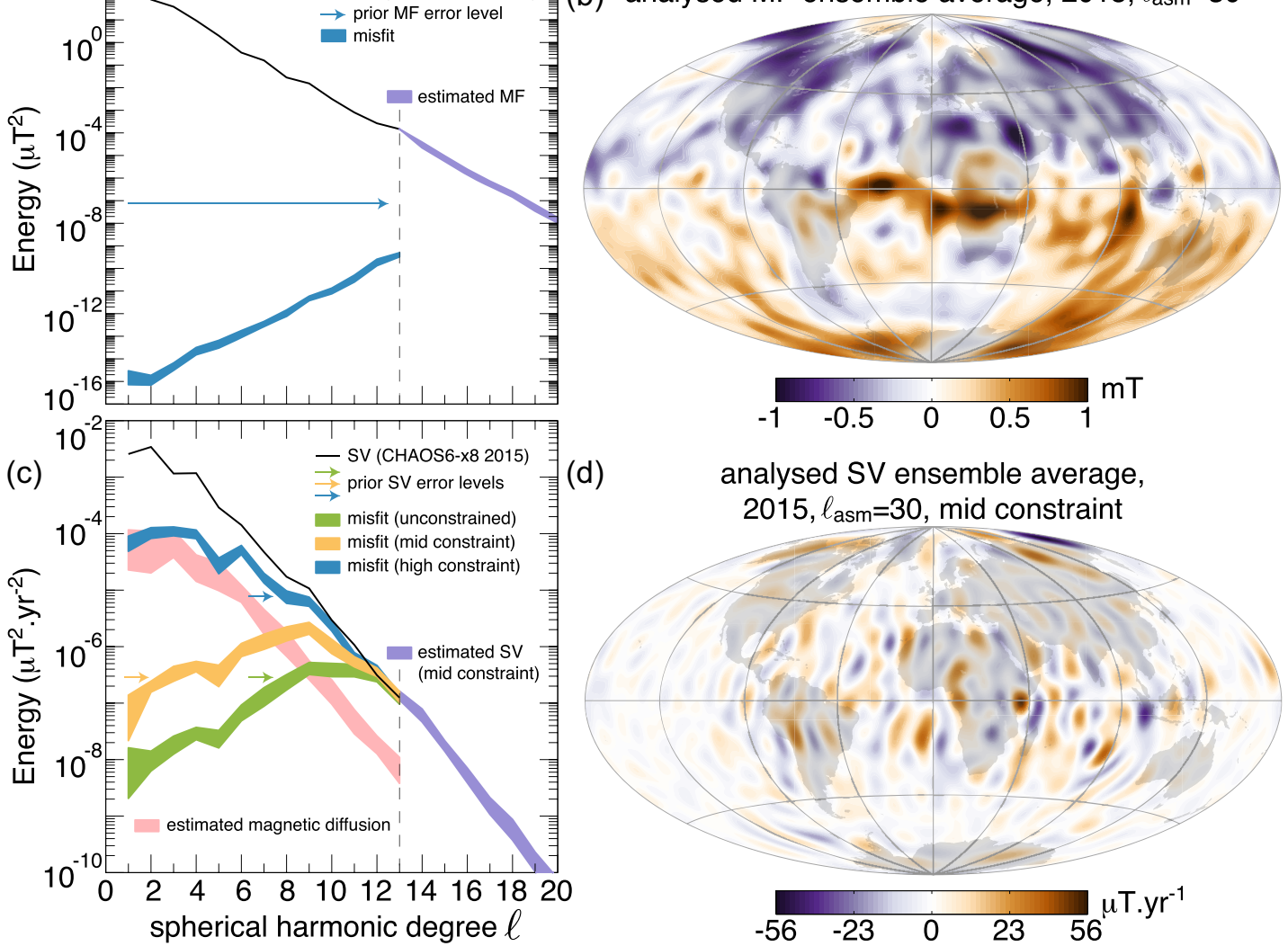

(d)
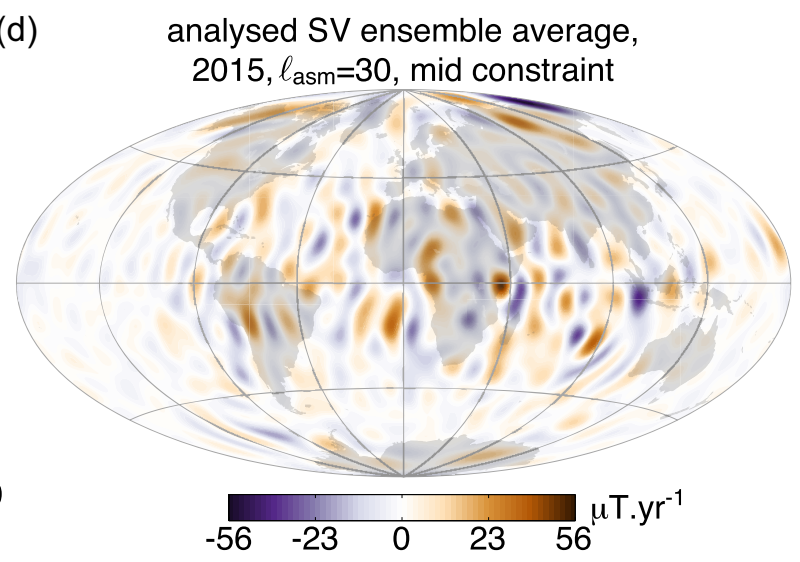

Figure 1. (a, c) Main field (MF) and secular variation (SV) characteristics of ensembles of core states inferred from CHAOS-6x8 data in 2015. Mauersberger (a) and Lowes (c) energy spectra of the MF and SV, showing the data (black lines), the level of error specified prior to the inversion (arrows), the estimated \pm 1 $S D$ range of diffusion within the ensemble of inverted states (pink shaded region), the range of fit residuals (blue, yellow, green shaded regions) obtained in inversions with varying levels of the QG-MAC constraint (note that analysis of MF is independent on this constraint), and the range of small scales estimated from the data (violet shaded regions). (b, d) Hammer projections of the ensemble averaged radial magnetic field (b) and radial secular variation (d) at the core-mantle boundary (orange is outwards) obtained in 2015, featuring small-scale details between degrees $\ell_{\mathrm{obs}}=13$ and $\ell_{\text {asm }}=30$ estimated through the inversion procedure.

$\mathbf{b}=\left[W_{\ell}^{m}\left(r_{j}\right), Z_{\ell}^{m}\left(r_{j}\right)\right]^{\top}, 1 \leq j \leq N R, 1 \leq \ell \leq \ell_{\mathrm{asm}}$,

$\mathbf{x}=\left[S_{\ell}^{m}\left(r_{j}\right), T_{\ell}^{m}\left(r_{j}\right), C_{\ell}^{m}\left(r_{j}\right)\right]^{\top}, 1 \leq j \leq N R, 0 \leq \ell \leq \ell_{\mathrm{asm}}$.

Here the spheroidal flow potential $S_{\ell}^{m}=\mathrm{d}\left(r P_{\ell}^{m}\right) / r \mathrm{~d} r$ is preferred over the poloidal flow potential $P_{\ell}^{m}$, and $\ell_{\text {asm }}=30$ is the maximum degree at which the analysis of the core state is performed. The variability of hidden core state quantities, as well as the general covariance properties of core state variables is represented through an ensemble of $N=746$ decorrelated samples $\mathbf{x}_{i}, \mathbf{b}_{i}$ obtained during a preliminary free run of the coupled Earth dynamo model spanning about $70000 \mathrm{yr}$. The ensemble average is defined as (e.g. in the case of $\mathbf{x}_{i}$ )

$\overline{\mathbf{x}}=\frac{1}{N} \sum_{i=1}^{N} \mathbf{x}_{i}$,

and the covariance matrices $P, Q$ for $\mathbf{b}, \mathbf{x}$ are approximated by

$\mathbf{P} \approx \frac{1}{N-1} \sum_{i=1}^{N}\left(\left(\mathbf{b}_{i}-\overline{\mathbf{b}}\right)\left(\mathbf{b}_{i}-\overline{\mathbf{b}}\right)^{\prime}\right)$,
$\mathbf{Q} \approx \frac{1}{N-1} \sum_{i=1}^{N}\left(\left(\mathbf{x}_{i}-\overline{\mathbf{x}}\right)\left(\mathbf{x}_{i}-\overline{\mathbf{x}}\right)^{\prime}\right)$.

These matrices essentially have a block-diagonal shape (see examples in fig. 3 of Aubert 2013) that couples adjacent spherical harmonic degrees within an harmonic order. As in Aubert (2015) the inversion for a complete core state proceeds in two steps. The first step remains an ensemble-based statistical inference of the magnetic field throughout the core from the data vector $\mathbf{d}$. An observation operator $\mathbf{H}$ is introduced, with this matrix having a simple form consisting of ones in entries corresponding to an observed quantity (the poloidal magnetic field at the core surface), and zeros otherwise. The direct problem then writes $\mathbf{H b}=\mathbf{d}$, and an ensemble of inverse (or analysed) solutions $\mathbf{b}_{i}^{a}$ is obtained from the initial states $\mathbf{b}_{i}$ through a classical Bayesian inference (Aubert 2015):

$\mathbf{b}_{i}^{a}=\mathbf{b}_{i}+\frac{\mathbf{P H}^{\prime}}{\mathbf{H P H}+\mathbf{R}}\left(\mathbf{d}-\mathbf{H b}_{i}\right)$.

From the observable magnetic field, the ensemble $\mathbf{b}_{i}^{a}$ estimates the variability of the magnetic field hidden inside the core, including its toroidal potential and its small-scale content up to degree $\ell_{\text {asm }}$ (see Fig. 1b). As is generally the case in an ensemble Kalman filter (Evensen 2003), the average value $\overline{\mathbf{b}_{i}^{a}}$ provides the best estimator 


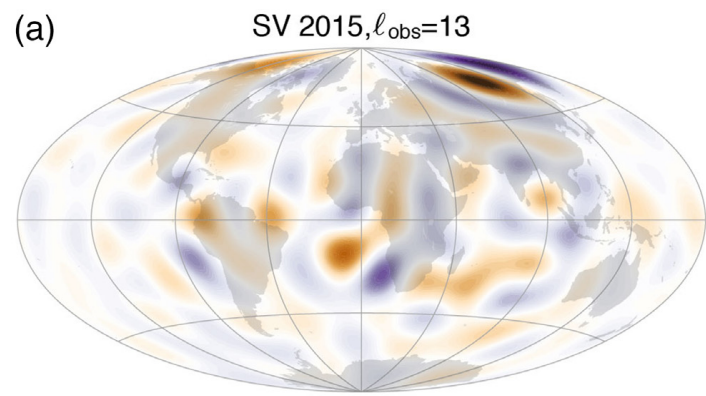

(b) ensemble averaged residual, no constraint

(c) ensemble averaged residual, mid constraint
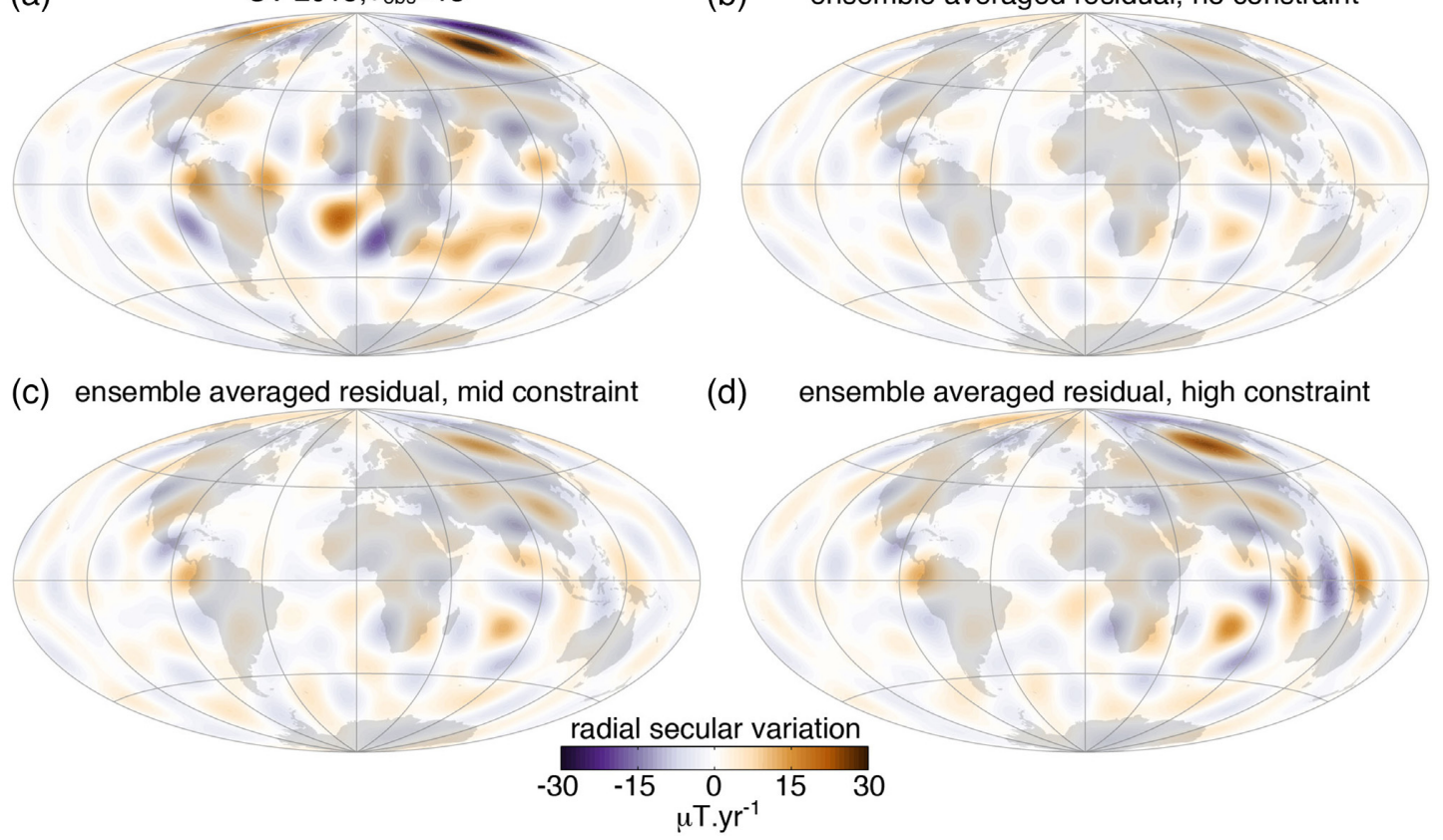

(d) ensemble averaged residual, high constraint

Figure 2. (a) Hammer projection of the core-mantle boundary radial secular variation from CHAOS-6x8 in 2015 , presented up to spherical harmonic degree $\ell_{\mathrm{obs}}=13$. (b-d) Ensemble averaged residuals of the fit to SV data obtained in the unconstrained (b), mid-constrained (c) and highly constrained (d) inversions, presented at the same truncation level.

given the data, the dynamo model, and their respective covariance properties. As eq. (22) produces an estimate of magnetic field throughout the core, and in particular below the core surface, it is possible to compute an ensemble of estimates for the core surface magnetic diffusion and Lorentz force, which we, respectively, represent through the vectors $\boldsymbol{\delta}_{i}$ and $\boldsymbol{\lambda}_{i}$ as

$\boldsymbol{\delta}_{i}=\eta\left[\left(\nabla^{2} W_{i}^{a}\right)_{\ell}^{m}\right]^{\top}, r=r_{o}, 1 \leq \ell \leq \ell_{\mathrm{asm}}$

$\lambda_{i}=\left(\begin{array}{c}{\left[\left(\mathbf{r} \cdot \nabla \times \mathbf{f}_{i}^{a}\right)_{\ell}^{m}\right]^{\top}} \\ {\left[\left(\mathbf{r} \cdot \nabla \times \nabla \times \mathbf{f}_{i}^{a}\right)_{\ell}^{m}\right]^{\top}}\end{array}\right), r=r_{o}, 1 \leq \ell \leq \ell_{\mathrm{asm}}$.

Here $W_{i}^{a}$ and $\mathbf{f}_{i}^{a}$ are, respectively, the poloidal magnetic potential and Lorentz force computed from $\mathbf{b}_{i}^{a}$. This latter force is evaluated by transforming $\mathbf{b}_{i}^{a}$ in the physical space to yield the magnetic field vector $\mathbf{B}_{i}^{a}$ and its curl, and then evaluating the product $\mathbf{f}_{i}^{a}=\left(\nabla \times \mathbf{B}_{i}^{a}\right) \times \mathbf{B}_{i}^{a} / \rho \mu_{0}$ over the discrete physical grid.

In a second step, we introduce the ensemble of model operators $\mathbf{M}_{i}$ linking the hydrodynamic state vector $\mathbf{x}$ to the secular variation data $\dot{\mathbf{d}}$ as well as incorporating the QG-MAC constraint,

$\mathbf{M}_{i}=\left(\begin{array}{c}\mathbf{M}_{i}^{\mathrm{SV}} \\ \mathbf{M}^{\mathrm{MAC}}\end{array}\right)$,

as well as the ensemble of direct problems

$\mathbf{M}_{i} \mathbf{x}=\left(\begin{array}{c}\dot{\mathbf{d}}-\boldsymbol{\delta}_{i} \\ -\lambda_{i}\end{array}\right)$

As already detailed in Aubert (2015), the matrices $\mathbf{M}_{i}^{\mathrm{SV}}$ encapsulate the spectral representations of the magnetic induction term $\nabla \times\left(\mathbf{u} \times \mathbf{B}_{i}^{a}\right)$ from eq. (2), taken at the core surface $\left(r=r_{o}\right)$. Its coefficients therefore involve the analysed magnetic field $\mathbf{b}_{i}^{a}\left(r_{o}\right)$, including its small scales up to degree $\ell_{\text {asm }}$, together with classical Elsasser and Adams-Gaunt coupling integrals (see Aubert 2013). The ensemble of these matrices samples the variability of induction that results from the variability of hidden core surface magnetic field small scales. This is the procedure through which the inverse framework handles the classical truncation errors inherent to the core flow inversion problem (e.g. Eymin \& Hulot 2005).

The matrix $\mathbf{M}^{\mathrm{MAC}}$ encapsulates the linear part of the QG-MAC constraint at the core surface, also written in the spectral space. To find its coefficients, we consider the radial components of the curl and double curl of eq. (1) at $r=r_{o}$, neglect inertia and viscosity and project on the spherical harmonic basis at degree and order $\ell$, $m$ (see e.g. Dormy 1997, eq. 2.102):

$$
\begin{aligned}
& 2 \Omega\left[i m T_{\ell}^{m}\left(r_{o}\right)\right] \\
& -2 \Omega\left[K_{12} S_{\ell-1}^{m}\left(r_{o}\right)+K_{22} S_{\ell+1}^{m}\left(r_{o}\right)\right] \\
& =-\frac{1}{\rho \mu_{0}}[\mathbf{r} \cdot \nabla \times((\nabla \times \mathbf{B}) \times \mathbf{B})]_{\ell}^{m}\left(r_{o}\right), \\
& -4 \frac{\Omega}{r_{o}}\left[i m S_{\ell}^{m}\left(r_{o}\right)\right] \\
& -2 \frac{\Omega}{r_{o}}\left[\left(K_{11}+K_{12}\right) T_{\ell-1}^{m}\left(r_{o}\right)+\left(K_{21}+K_{22}\right) T_{\ell+1}^{m}\left(r_{o}\right)\right] \\
& +\ell(\ell+1) \frac{g_{o}}{\rho r_{o}} C_{l}^{m}\left(r_{o}\right) \\
& =-\frac{1}{\rho \mu_{0}}[\mathbf{r} \cdot \nabla \times \nabla \times((\nabla \times \mathbf{B}) \times \mathbf{B})]_{\ell}^{m}\left(r_{o}\right) .
\end{aligned}
$$

Eq. (27) refers to the toroidal part of the QG-MAC balance, and eq. (28) represents its spheroidal (or poloidal) part. Note that the derivation of $(27,28)$ from eq. (2.102) in Dormy (1997) involves the following relationships, which stem from the stress-free conditions at the core-mantle boundary:

$P_{\ell}^{m}\left(r_{o}\right)=0$,

$\nabla^{2} P_{\ell}^{m}\left(r_{o}\right)=2 S_{\ell}^{m}\left(r_{o}\right) / r_{o}$, 


$$
\begin{aligned}
\frac{\mathrm{d} P_{\ell}^{m}}{\mathrm{~d} r}\left(r_{o}\right) & =S_{\ell}^{m}\left(r_{o}\right), \\
\frac{\mathrm{d} T_{\ell}^{m}}{\mathrm{~d} r}\left(r_{o}\right) & =T_{\ell}^{m}\left(r_{o}\right) / r_{o} .
\end{aligned}
$$

The coupling coefficients $K_{11}, K_{12}, K_{21}, K_{22}$ write

$K_{11}=(\ell+1)(\ell-1)^{2} \sqrt{\frac{(\ell-m)(\ell+m)}{(2 \ell-1)(2 \ell+1)}}$,

$K_{12}=-K_{11} /(\ell-1)$,

$K_{21}=-\ell(\ell+2)^{2} \sqrt{\frac{(\ell+m+1)(\ell-m+1)}{(2 \ell+3)(2 \ell+1)}}$,

$K_{22}=K_{21} /(\ell+2)$.

Note also that these last expressions account for the omission of spherical harmonic normalization constants in eq. (2.101) of Dormy (1997) (see Errata of this document). The route to the present expressions therefore involves reintroducing these constants (as given by his eq. 2.82), writing the corrected equation for degrees $\ell+1$ and $\ell-1$ and collecting the terms relevant to degree $\ell$. Finally, the coefficients acting on $T, S, C$ in the left-hand side of $(27,28)$ are collected to build $\mathbf{M}^{\mathrm{MAC}}$, while the non-linear Lorentz force terms in the right-hand side are treated explicity as the $-\boldsymbol{\lambda}_{i}$ contribution in eq. (26).

At this point, the ensemble of direct problems given by (26) relate the hydrodynamic state vector $\mathbf{x}$ to the secular variation data $\dot{\mathbf{d}}$ through the joint consideration of the core surface QG-MAC balance in (1) and the full magnetic induction eq. (2), including magnetic diffusion. Similarly to Aubert (2015), an ensemble of inverse solutions $\mathbf{x}_{i}^{a}$ is again obtained by Bayesian inference:

$$
\mathbf{x}_{i}^{a}=\mathbf{x}_{i}+\frac{\mathbf{Q} \mathbf{M}_{i}^{\prime}}{\mathbf{M}_{i} \mathbf{Q} \mathbf{M}_{i}^{\prime}+\left(\begin{array}{ll}
\dot{\mathbf{R}} & \mathbf{0} \\
\mathbf{0} & \Sigma
\end{array}\right)}\left[\left(\begin{array}{c}
\dot{\mathbf{d}}-\boldsymbol{\delta}_{i} \\
-\lambda_{i}
\end{array}\right)-\mathbf{M}_{i} \mathbf{x}_{i}\right] .
$$

This ensemble describes the variability of core flows that account for the secular variation data while respecting the QG-MAC constraint, given the uncertainty on the core magnetic field below the core surface and at small unobservable scales. The average value $\overline{\mathbf{x}_{i}^{a}}$ again provides the best estimator of $\mathbf{x}$ given the geomagnetic data, the dynamo model, their respective covariance properties and the dynamical constraint. The diagonal matrix $\Sigma$ represents the tolerance level for the enforcement of the QG-MAC balance. For the toroidal and spheroidal parts of $\boldsymbol{\Sigma}$, respectively corresponding to eqs $(27,28)$, we adopt the following coefficients corresponding to an even distribution of the constraint level among spherical harmonic degrees and orders:

$\boldsymbol{\Sigma}=\left(\begin{array}{cc}\mathbf{L}_{\mathbf{2}} \sigma_{T}^{2} & \mathbf{0} \\ \mathbf{0} & \mathbf{L}_{\mathbf{2}} \sigma_{S}^{2}\end{array}\right)$.

Here $\mathbf{L}_{2}$ is the diagonal matrix with coefficients $\ell(\ell+1)$. To guide the choice of $\sigma_{T}, \sigma_{S}$, it is useful to relate these coefficients to the typical r.m.s level of flow acceleration $<\partial_{t} \mathbf{u}\left(r_{o}\right)>$ that is expected from the deviation to the QG-MAC balance. Imbalances in eqs $(27,28)$ predominantly correspond to inertial contributions (see Fig. 3) and from eq. (1) they write $\ell(\ell+1)[\mathbf{r} \cdot \nabla \times \mathrm{Du} / \mathrm{D} t]_{\ell}^{m}$ and $\ell(\ell+1)[\mathbf{r} \cdot \nabla \times \nabla \times \mathrm{Du} / \mathrm{D} t]_{\ell}^{m}$, respectively. The presence of a total derivative precludes an exact relationship but the following approximate relationship holds:

$$
<\partial_{t} \mathbf{u}\left(r_{o}\right)>\approx \sqrt{N_{30}\left(\sigma_{T}^{2}+r_{S}^{2} \sigma_{S}^{2}\right)} .
$$

Here the angled brackets denote the root-mean-squared surface average, $N_{30}=496$ is the number of complex spherical harmonic coefficients involved up to degree $\ell_{\text {asm }}=30$ and $r_{S}=0.03 D$ is a typical length scale for the radial variations of $S$ near the coremantle boundary in the coupled earth model. We target Earthlike flow accelerations of $\left\langle\partial_{t} \mathbf{u}\left(r_{o}\right)>\approx 1 \mathrm{~km} \mathrm{yr}^{-2}\right.$ (Aubert 2014). Guided by eq. (39), in our moderately constrained case we adopt $\sigma_{T}=2.410^{-2} \mathrm{~km} \mathrm{yr}^{-2}$ and $\sigma_{S}=6.410^{-4} 1 \mathrm{yr}^{-2}$. As will be seen in Fig. 3, this value of $\sigma_{T}$ suffices to reduce the toroidal flow acceleration of the inverse solutions to the levels commonly observed in a free run of the coupled earth model. In an attempt to also reduce the spheroidal flow acceleration to such levels, in our highly constrained case we keep $\sigma_{T}=2.4 \times 10^{-2} \mathrm{~km} \mathrm{yr}^{-2}$ but reduce $\sigma_{S}$ to $3.2 \times 10^{-4} 1 \mathrm{yr}^{-2}$. These values are summarized in Table 1 together with the corresponding level of data error in the three inversion cases (no constraint, mid constraint, high constraint) that we analyse.

At this point we have completed the procedure through which an ensemble of analysed complete core states $\mathbf{x}_{i}^{a}, \mathbf{b}_{i}^{a}$ can be constructed, that accounts for the core surface magnetic field and secular variation data at a given epoch while respecting the QG-MAC constraint at the core surface. The ensemble average estimates the most probable state given the data, the dynamo model, their respective covariances and the dynamical constraint. The variability within the ensemble accounts for the uncertainty in the variables that are hidden from observation (at depth in the core or at small scales).

\subsection{Evaluation of misfits}

For each ensemble member and at each given epoch, we use six misfit quantities to characterize the deviation of inverse solutions from the data, from the behaviour of the prior numerical model, and from the imposed constraints. Among these, five normalized quantities are constructed such that a unit value represents one standard deviation of the distribution of data errors or model variability. First, we introduce two quantities measuring the deviation to the main field (MF) and secular variation (SV) data, normalized by the respective levels of observation errors $\epsilon_{B}$ and $\epsilon_{\dot{B}}$ :

$$
\begin{aligned}
\Delta_{\mathrm{MF}}(i) & =\sqrt{\frac{1}{N_{13}}\left(\mathbf{d}-\mathbf{H b}_{i}^{a}\right)^{\prime} \mathbf{R}^{-1}\left(\mathbf{d}-\mathbf{H b}_{i}^{a}\right)}, \\
\Delta_{\mathrm{SV}}(i) & =\sqrt{\frac{1}{N_{13}}\left(\dot{\mathbf{d}}-\boldsymbol{\delta}_{i}-\mathbf{M}_{i}^{S V} \mathbf{x}_{i}^{a}\right)^{\prime} \dot{\mathbf{R}}^{-1}\left(\dot{\mathbf{d}}-\boldsymbol{\delta}_{i}-\mathbf{M}_{i}^{S V} \mathbf{x}_{i}^{a}\right) .}
\end{aligned}
$$

Here $N_{13}=105$ is the number of complex spherical harmonic coefficients involved in a decomposition up to degree and order $\ell_{\mathrm{obs}}$ $=13$.

Secondly, we normalize the amplitude of analysed state vector increments with the variability of the prior numerical model, leading to the three quantities

$$
\begin{aligned}
& \Delta_{B}(i)=\sqrt{\frac{1}{N_{30}}\left(\mathbf{b}_{i}^{a}-\mathbf{b}_{i}\right)_{B\left(r_{o}\right)}^{\prime} \mathbf{P}_{B\left(r_{o}\right)}^{-1}\left(\mathbf{b}_{i}^{a}-\mathbf{b}_{i}\right)_{B\left(r_{o}\right)}}, \\
& \Delta_{u}(i)=\sqrt{\frac{1}{2 N_{30}}\left(\mathbf{x}_{i}^{a}-\mathbf{x}_{i}\right)_{u\left(r_{o}\right)}^{\prime} \mathbf{Q}_{u\left(r_{o}\right)}^{-1}\left(\mathbf{x}_{i}^{a}-\mathbf{x}_{i}\right)_{u\left(r_{o}\right)}}, \\
& \Delta_{C}(i)=\sqrt{\frac{1}{N_{30}}\left(\mathbf{x}_{i}^{a}-\mathbf{x}_{i}\right)_{C\left(r_{o}\right)}^{\prime} \mathbf{Q}_{C\left(r_{o}\right)}^{-1}\left(\mathbf{x}_{i}^{a}-\mathbf{x}_{i}\right)_{C\left(r_{o}\right)}} .
\end{aligned}
$$




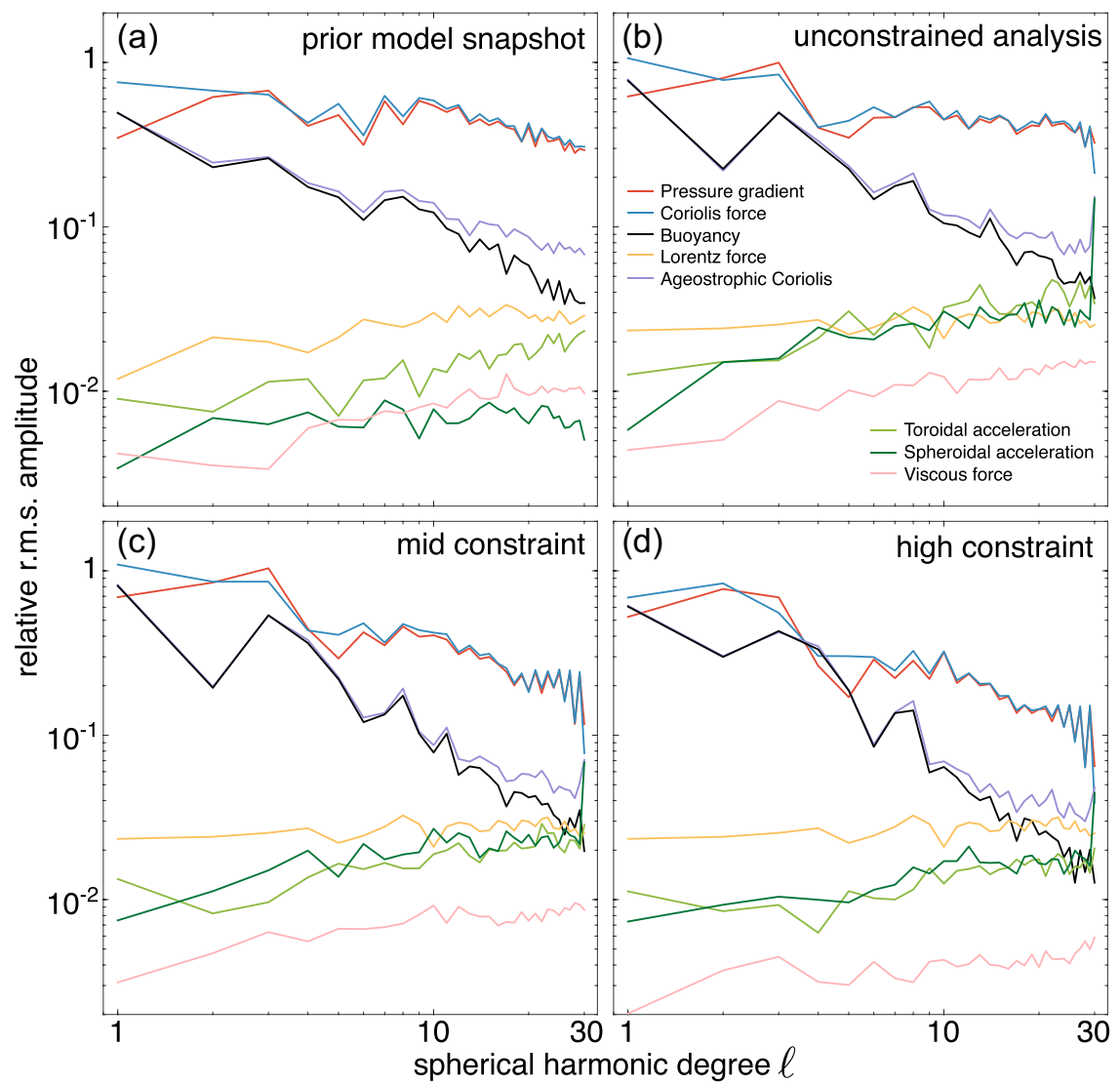

Figure 3. Scale-dependent force balance diagrams at the core surface for: (a) a snapshot obtained during a free run of the numerical dynamo prior model, and (b-d) individual ensemble members obtained from inversion of CHAOS-6x8 data in 2010 with varying levels of the imposed QG-MAC constraint. These ensemble members are numerically integrated forward in time with the dynamo model to obtain the instantaneous force balance immediately after the analysis epoch. R.m.s amplitudes of each force present in eq. (1) are represented as functions of the spherical harmonic degree $\ell$ (see Aubert et al. 2017, for technical computation details). Forces are normalized relative to the maximum value of the pressure force. The ageostrophic Coriolis force represents the residual Coriolis force after removal of the pressure force. Inertial forces are represented through separate toroidal and spheroidal contributions to $\partial_{t} \mathbf{u}$.

Table 1. Values of data error (Section 2.2) and QG-MAC constraint level (Section 2.3) parameters used in the inversions.

\begin{tabular}{lcccc}
\hline & $\epsilon_{B}(\mathrm{nT})$ & $\epsilon_{\dot{B}}\left(\mathrm{nT} \mathrm{yr}^{-1}\right)$ & $\sigma_{T}\left(\mathrm{~km} \mathrm{yr}^{-2}\right)$ & $\sigma_{S}\left(1 \mathrm{yr}^{-2}\right)$ \\
\hline Unconstrained & 1 & 2 & - & - \\
Mid constraint & 1 & 2 & $2.4 \times 10^{-2}$ & $6.4 \times 10^{-4}$ \\
High constraint & 1 & 10 & $2.4 \times 10^{-2}$ & $3.2 \times 10^{-4}$ \\
\hline
\end{tabular}

Here subscripts $B\left(r_{o}\right), u\left(r_{o}\right), C\left(r_{o}\right)$ indicate that the computation is restricted to the state vector and covariance matrix components, respectively, referring to the poloidal magnetic field, toroidal and spheroidal flow, and density anomaly at the core surface. Finally, we characterize the degree of enforcement of the QG-MAC balance through an equivalent core surface flow acceleration timescale

$\tau_{\text {flow }}(i)=\frac{<\mathbf{u}_{i}^{a}\left(r_{o}\right)>}{<\partial_{t} \mathbf{u}_{i}^{a}\left(r_{o}\right)>}$.

Here $\mathbf{u}_{i}^{a}\left(r_{o}\right)$ is the analysed core flow described by the state vector $\mathbf{x}_{i}^{a}$ and we recall that the angled brackets denote the root-meansquared surface average. Along the same lines as those introduced for eq. (39), the r.m.s. core surface flow acceleration $\left\langle\partial_{t} \mathbf{u}_{i}^{a}\left(r_{o}\right)>\right.$ that is effectively obtained after the inversion can be related to the residuals of the QG-MAC balance enforcement through the following approximate relationship:

$$
\begin{aligned}
& <\partial_{t} \mathbf{u}_{i}^{a}\left(r_{o}\right)>^{2} \approx \\
& \left(\mathbf{M}^{\mathrm{MAC}} \mathbf{x}_{i}^{a}+\lambda_{i}\right)_{\text {tor }}^{\prime} \mathbf{L}_{2}{ }^{-1}\left(\mathbf{M}^{\mathrm{MAC}} \mathbf{x}_{i}^{a}+\lambda_{i}\right)_{\text {tor }} \\
& +r_{S}^{2}\left(\mathbf{M}^{\mathrm{MAC}} \mathbf{x}_{i}^{a}+\lambda_{i}\right)_{\text {spher }}^{\prime} \mathbf{L}_{\mathbf{2}}^{-1}\left(\mathbf{M}^{\mathrm{MAC}} \mathbf{x}_{i}^{a}+\lambda_{i}\right)_{\text {spher }},
\end{aligned}
$$

where the typical length scale $r_{S}$ for the radial variations of $S$ near the core-mantle boundary is again involved, and the subscripts denote the toroidal and spheroidal parts of the vector $\mathbf{M}^{\mathrm{MAC}} \mathbf{x}_{i}^{a}+\lambda_{i}$.

\subsection{Ensemble subset and forecasts}

The matrices $\mathbf{P}$ and $\mathbf{Q}$ are computed only once for all inversions, but given the large size $N$ of the ensemble and the yearly intervals at which the geomagnetic field model is analysed, it is computationally expensive to retain all ensemble members for the analysis and the subsequent forecasts. For all epochs, the same subset of 21 ensemble members is therefore retained throughout Section 3. 
In particular, in Section 3.4 these are used as starting points for the temporal integration of the numerical model, thereby providing a forecast for the future, with the ensemble mean representing the most likely forecast and the ensemble variance accounting for the forecast uncertainty. Here the selection of ensemble members is made on the basis of the quality of their forecast of epoch 2015 from epoch 2009. This optimizes the forecasts performed throughout the epoch range 1999-2019 (a desirable property in an operational perspective) while otherwise producing results that are reasonably converged against doubling of the ensemble size.

As in a previous study (Fournier et al. 2015), a 'dynamic' forecast strategy based on the full set of eqs (1)-(3) will be used in Section 3.4, together with a 'steady flow' strategy that only involves the numerical integration of eq. (2). In the steady flow configuration, the magnetic acceleration therefore only comprises the part that couples the magnetic variation to the flow and the contribution of diffusive acceleration (see Aubert 2018, and Section 3.4 for a description of contributions to the magnetic acceleration). In the dynamic configuration, the magnetic acceleration is completed with the part that couples the magnetic field to the flow acceleration. The resulting forecasts are similar in methodology to previous attempts to forecast geomagnetic variations by using steady (Beggan \& Whaler 2010) or accelerating (Whaler \& Beggan 2015) core flows, with the notable difference that diffusive acceleration is presently accounted for.

\section{RESULTS}

\subsection{Fit to data and adequation with prior numerical dynamo model}

Table 2 and Figs 1 and 2 characterize the fit quality of our ensembles of inverse solutions to the MF and SV data, as well as their level of compatibility with the prior numerical dynamo model. The inversion misfit to MF data is generally excellent, as shown by the low residuals (Fig. 1a) well below the specified observation error level $\epsilon_{B}=1 \mathrm{nT}$ which is evenly distributed among spherical harmonic degrees (the corresponding flat spectrum being marked by an arrow in Fig. 1a). As a consequence, the normalized misfit to the data $\Delta_{\mathrm{MF}}$ is also very low (Table 2). The normalized deviation to the prior model variability $\Delta_{B}=1.8$ obtained throughout the considered epoch range also indicates that the MF prescribed by the numerical dynamo model is also reasonably compatible with that required by the data. This compatibility can also be seen in the estimation of unobserved MF small scales made by the inversion scheme, where the transition between observed $\left(\ell \leq \ell_{\text {obs }}\right.$, black line in Fig. 1a) and estimated $\left(\ell_{\mathrm{obs}}<\ell \leq \ell_{\text {asm }}\right.$, purple \pm 1 std. dev. range) scales is seamless, both in amplitude and in spectral slope. The resulting ensemble-averaged, analysed radial magnetic field at the core-mantle boundary (Fig. 1b) featuring observed and estimated scales is broadly consistent with images obtained through earlier attempts to obtain high-resolution core MF models (see e.g. fig. 5 of Finlay et al. 2012). The level of magnetic diffusion computed from the analysed MF (pink range in Fig. 1c) and involved in the inversion of eq. (2) is also broadly consistent with that found in unconstrained runs of the dynamo model (Aubert 2013).

In our three (unconstrained, mid-constrained and highly constrained) cases, the misfit to the SV data (green, yellow and blue spectra in Fig. 1c) is generally higher than the observation error that is prescribed (the corresponding flat spectrum levels of which are marked by green, yellow and blue arrows in Fig. 1c), leading to $\Delta_{\mathrm{SV}}$ $>1$. This is especially true in cases where the QG-MAC constraint is imposed, which logically leads to a degradation of the fit to SV data since adding constraints to the inverse problem (26) necessarily shifts the trade-off between fit quality and adherence to the constraints. Still, values of $\Delta_{\mathrm{SV}}$ remain below 2 standard deviations, and the fit residuals to SV remain weak relative to the data for $\ell \leq 9$ (Figs 1c, 2b and c) except in the highly constrained case where the observation error level $\epsilon_{\dot{B}}$ on the SV has been increased on purpose in order to best satisfy the QG-MAC constraint (see Table 1). In this case (Fig. 2d), strong SV misfits at the core-mantle boundary are essentially located in a longitudinal band between $90^{\circ} \mathrm{E}$ and $180^{\circ} \mathrm{E}$. The compatibility between the flow and density anomaly fields prescribed by the numerical model and those required by the data is also fair, with values of the normalized deviations to the prior model variability $\Delta_{u} \approx 1$ and $\Delta_{C} \approx 2$. As was the case with the MF, this compatibility can also be seen in the seamless continuation of the observed SV energy spectrum $\left(\ell \leq \ell_{\text {obs }}\right.$, black line in Fig. 1c corresponding to the pattern in Fig. $2 \mathrm{a})$ into the estimated part $\left(\ell_{\mathrm{obs}}\right.$ $<\ell \leq \ell_{\text {asm }}$, purple range in Fig. 1c). The corresponding ensemble averaged, analysed radial secular variation at the core-mantle boundary (Fig. 1d) features axially elongated small-scale structures which are typical of induction by an axially columnar flow, as also usually observed in the prior numerical dynamo model.

\subsection{Core surface force balance}

To examine the force balance that is realized in the analysed core states, we rely on the scale-dependent representation introduced and discussed in our previous studies, where the r.m.s. amplitude of each force is represented as a function of the spherical harmonic degree $\ell$. In Fig. 3, we focus specifically on the force balance at the core surface, as this is the location where we can check the enforcement of the QG-MAC constraint specified in our inversions through eqs (27) and (28). The amplitude hierarchy of forces found at the surface in a free run snapshot of the coupled earth model (Fig. 3a) conforms to results previously obtained in the bulk (Aubert et al. 2017; Aubert 2019; Schwaiger et al. 2019). The leading-order force balance is a QG equilibrium between the Coriolis force and the pressure gradient. At the next order in amplitude, a MAC equilibrium is found between the Lorentz, buoyancy and ageostrophic Coriolis force. The main difference between surface and interior is that the magnetic field being weaker at the core surface, the crossing between the buoyancy and Lorentz force lines occurs at rather small scales $\ell$ $=30$ and beyond, while it occurs at $\ell \approx 10$ in the bulk. Inertial and viscous forces can be interpreted as the residual of the QG-MAC balance, and Fig. 3(a) shows that this residual is much lower than the contribution from buoyancy but not significantly smaller than that of the Lorentz force, a situation which is typical of the start of the parameter space path where the coupled earth model is located (Aubert et al. 2017).

Turning now to the results of inverse modelling, Fig. 3(b) shows that unconstrained inversions enforce the leading-order QG balance between Coriolis and pressure forces, with the deviations from this equilibrium being mainly balanced by the buoyancy force at large scales. Inertial and viscous contributions are also much lower than the buoyancy force except close to the analysis cut-off level $\ell_{\text {asm }}=$ 30. As previously highlighted in Aubert (2015), this thermal wind balance is indeed linear and therefore already well represented by the statistical cross correlations between $\mathbf{u}$ and $C$ present in matrix $\mathbf{Q}$ alone. However, the linear inversion framework cannot naturally handle the non-linear part of the QG-MAC balance, and the level of residuals is therefore as strong as the Lorentz force. In 
Table 2. Values of misfit quantities defined in Section 2.4, averaged over all yearly epochs between 1999.0 and 2019.0 and over ensemble members.

\begin{tabular}{lcccccc}
\hline & $\Delta_{\mathrm{MF}}$ & $\Delta_{\mathrm{SV}}$ & $\Delta_{B}$ & $\Delta_{u}$ & $\Delta_{C}$ & $\tau_{\text {flow }}(\mathrm{yr})$ \\
\hline Unconstrained & 0.03 & 1.1 & 1.8 & 0.8 & 1.8 & 7.8 \\
Mid constraint & 0.03 & 1.7 & 1.8 & 1.1 & 2.1 & 13.2 \\
High constraint & 0.03 & 1.7 & 1.8 & 1.0 & 1.8 & 17.5 \\
\hline
\end{tabular}

inversions where a moderate to high level of the constraint is imposed (Figs 3c and d), we verify that the residual level of inertial contributions move below the level of the Lorentz force, thereby better enforcing the QG-MAC balance. In the mid- and high constraint cases, inertial contributions have, respectively, decreased by 40 and 60 per cent relative to the unconstrained case, both values being consistent with the evolution of the diagnostic quantity $\tau_{\text {flow }}$ in Table 2. In the mid-constraint case (Fig. 3c) the residual level of toroidal inertia is already comparable to that of the prior, so it is not necessary to further decrease the constraint level $\sigma_{T}$ beyond this point. In contrast, it is significantly more difficult to enforce the spheroidal part of the constraint in order to obtain residuals of spheroidal inertia as low as those of the prior model. In particular, attempting to enforce high levels of this constraint leads to misfits to the SV (Fig. 2d) that are the signature of strong convective upwellings in the Eastern Hemisphere (see Fig. 4a). These upwellings are imposed more strongly by the prior model as the constraint level is raised, but they are not compatible with the SV data. As a consequence, imposing high levels of the QG-MAC constraint also affects the buoyancy field at small scales (see evolution of the buoyancy force line in Figs 3b, c and d) to mitigate the misfit to SV data.

\subsection{Core surface flow and underlying density anomaly structure}

As could be anticipated from the force balance structure presented in Fig. 3, enforcing the QG-MAC constraint in the inversion scheme does not significantly alter the resulting core surface flow structure. Fig. 4 indeed shows that constrained inversions present the same general circulation features as those already found by a number of authors (see list in Section 1). The general core surface flow pattern seen in an Atlantic-centred projection (Fig. 4a) is that of a double conveyor belt, with strong westward drift in the Atlantic hemisphere, convergence of higher-latitude core flow towards the equator together with upwelling from the deeper parts of the core beneath Indonesia, and divergence of equatorial core flow towards higher latitudes together with downwelling to the deeper core beneath central America. This is the surface signature of an eccentric axial columnar gyre found in the volume (see e.g. Pais \& Jault 2008; Aubert 2013; Gillet et al. 2015). The general circulation closes on itself in the Pacific hemisphere through a high-latitude jet (Figs $4 \mathrm{c}$ and e) that represents (at recent epochs) the strongest core flows that can be seen at the surface, locally accounting for the strongest core surface secular variation (Figs $2 \mathrm{a}$ and $4 \mathrm{~b}$ ). In the present inversions, while the jet shows symmetry with respect to the equator (compare Figs $4 \mathrm{c}$ and e), the southern hemisphere surface signature is weaker (since secular variation there is also weaker, Fig. 4d). This is in line with other inversions using prior information from numerical dynamos (e.g. Gillet et al. 2019) but departs from the pure axial invariance assumed in other studies (Pais \& Jault 2008; Gillet et al. 2015; Livermore et al. 2017). Unlike these last studies, also, we observe significant crossings of the axial cylinder tangent to the inner core (the tangent cylinder), and continuity between the jet outside the tangent cylinder with polar vortices inside the tangent cylinder. Such crossings of the tangent cylinder in rim-like, high-latitude core surface flows are routinely observed in numerical dynamo simulations results (Schaeffer et al. 2017; Aubert 2019). The evolution of the jet velocity over the period 1999-2019 is non-monotonous (Fig. 5) and only significant in the Northern hemisphere, with an average acceleration of $0.4 \mathrm{~km} \mathrm{yr}^{-2}$ there. This is in line with the results of Gillet et al. (2019) but more than four times smaller than the value reported by Livermore et al. (2017), despite a fit to the SV data which is locally as good (compare Figs 4c, e to their figs 3c, d). As in Gillet et al. (2019) we ascribe this difference to the handling of truncation errors and magnetic diffusion in our scheme, which is thereby safe against the risk of overfitting the SV.

Taking advantage of the controlled dynamical consistency of our constrained inversions, in Fig. 6 we next investigate the dominant driver of the core surface flow, with a focus on the high-latitude jet in the Pacific. As can be seen in Fig. 3, at the large scales imaged by the inversion the thermal wind driving (i.e. equilibrium between pressure, Coriolis and buoyancy forces) largely dominates the magnetic wind driving (where Lorentz force substitutes to the buoyancy force). Outside the tangent cylinder, the high-latitude jet observed in the Pacific hemisphere is in equilibrium with a longitudinally hemispheric pattern of buoyancy, especially visible in the Northern Hemisphere (Fig. 6a), with lighter fluid being present in the Atlantic hemisphere. Hemisphericity of the density anomaly field is built into the coupled Earth dynamo prior, but it has been previously shown that the density anomaly pattern obtained here is nevertheless largely data-driven (Aubert 2014). As is generally the case for thermal winds at high latitudes, the flow circulation is orthogonal to the density anomaly gradient and its amplitude is proportional to that of this gradient. The density anomaly gradients between high and low latitudes are stronger in the Pacific hemisphere than in the Atlantic (Fig. 6a), explaining the localized nature of the jet. Likewise, latitudinal density anomaly gradients are weaker in the Southern Hemisphere (Fig. 6b), accounting for the weaker jet there. Inside the tangent cylinder and in both hemispheres, polar vortices circulating around areas of lighter fluid locally merge with the highlatitude jet.

Comparing the core-originated variations of the length-of-day (1.o.d.) predicted by the ensemble of inverted core flows with geodetic observations provides another way to check the consistency of the discrete temporal sequence of inversions. Predicted 1.o.d. variations are found to be rather insensitive on the QG-MAC constraint level. In Fig. 7, we present the results obtained in the mid-constraint case. The difference between the predictions and a geodetic model generally lies at less than two standard deviations of the ensemble variability. This is a similar level of agreement, both in amplitude and trend, as that obtained in other recent studies (e.g. Aubert 2014; Gillet et al. 2015). This increases our confidence in the retrieval of $T_{1}^{0}\left(r_{o}\right)$ at the core surface and in the estimation of its internal profile $T_{1}^{0}(r)$. 

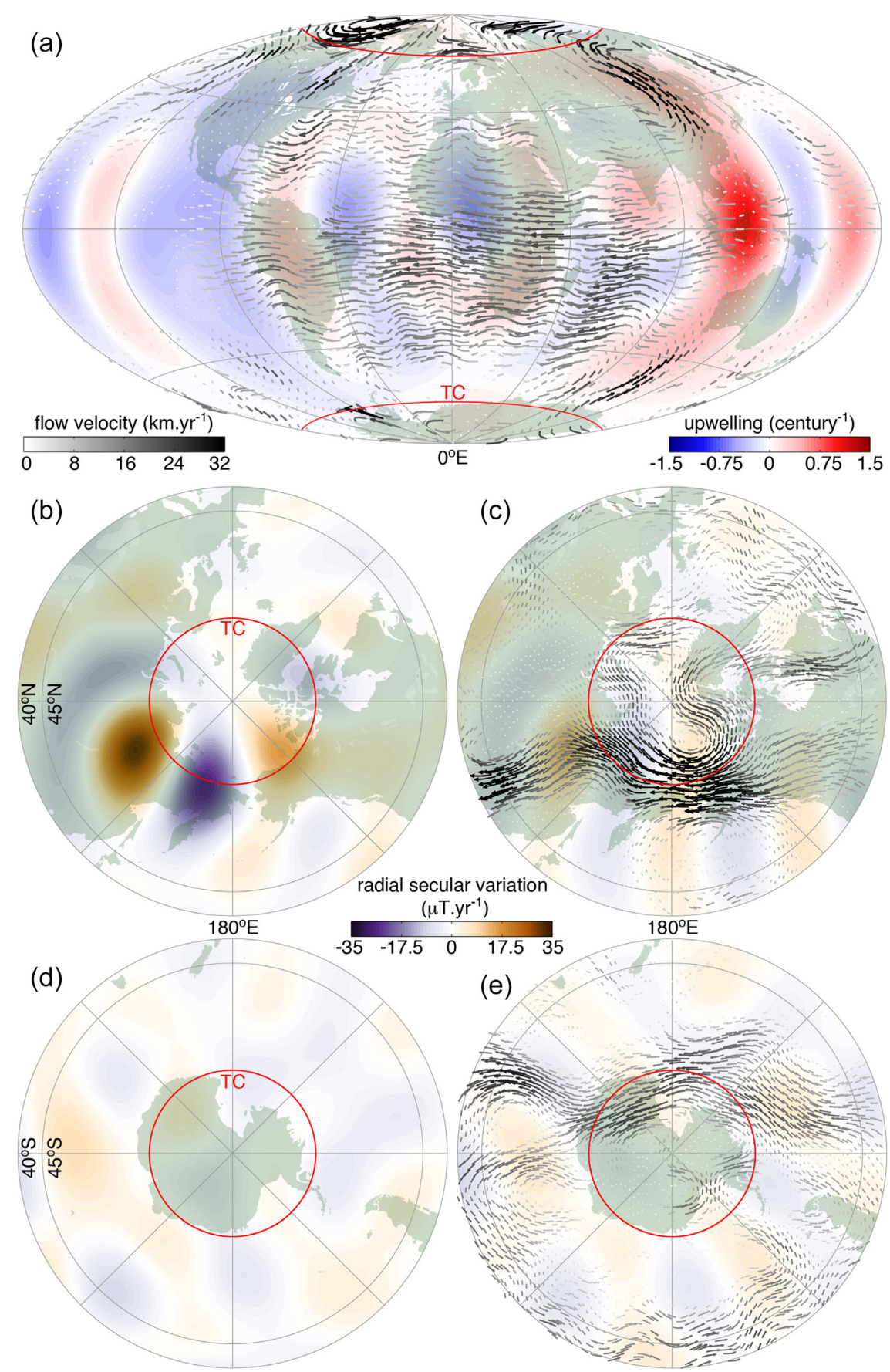

Figure 4. Visualizations of the ensemble averaged core surface flow (arrows) obtained in 2015 in the mid-constraint inversion case. Arrows are colour-coded with the local flow velocity $\left|\mathbf{u}\left(r_{o}\right)\right|$ (greyscale). (a) Hammer projection of core flow overplotted on the large-scale (up to degree 8) pattern of upwelling $-\partial_{r}(\mathbf{u} \cdot \mathbf{r})\left(r_{o}\right) / r_{o}$ (colour scale). (b, d) North (respectively South) polar projection of the core surface radial SV from CHAOS-6x8 in 2015 (same data as in Fig. 2a). (c, e) North (respectively South) polar projection of the core flow overplotted on the ensemble averaged fit residual to radial core surface SV (same data as in Fig. 2c).

\subsection{Short-term forecasts of the geomagnetic evolution}

We next examine the dynamical behaviour of our ensembles of core states, when using these as starting points for a time integration of the numerical model in order to predict the future evolution of the system. We restrict our experiments to the unconstrained and mid-constrained cases, as the highly constrained case does not fit the initial geomagnetic data with sufficient accuracy (Fig. 1c) to provide adequate forecasts. To illustrate the forecast quality, it is usually more relevant to represent the magnetic variation $\partial_{t} \mathbf{B}$ rather than the magnetic field $\mathbf{B}$ itself, as done in Figs 8 and 9 where the predicted geomagnetic evolution at 5- and 10-yr ranges is represented from a variety of starting points between epochs 1999 and 2014.

To assist the discussion of results, we first recall the geomagnetic acceleration equation obtained by taking the time derivative 


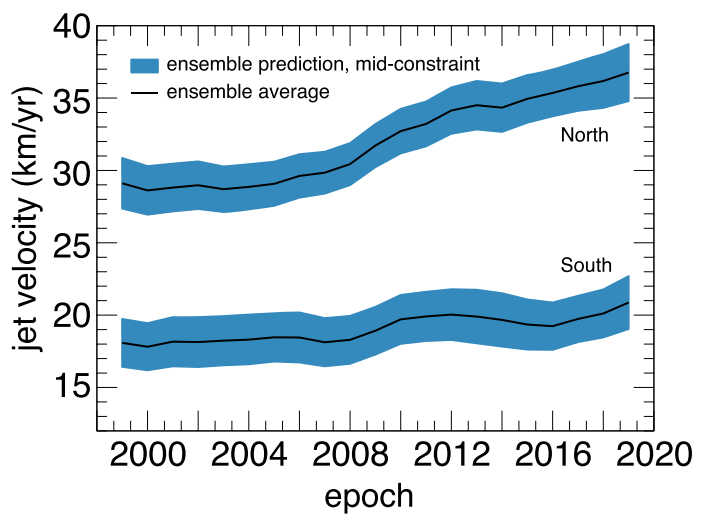

Figure 5. Evolution of the root-mean-squared velocity of the northern and southern parts of the high-latitude Pacific jet in the epoch range 1999-2019 (black line: ensemble average, blue shaded region: \pm 1 std. dev. range), in the mid-constraint inversion case. The jet velocity is determined by averaging $u^{2}\left(r_{o}\right)$ between longitudes $135^{\circ}-225^{\circ} \mathrm{E}$ and latitudes $63^{\circ}-77^{\circ}$ north or south.

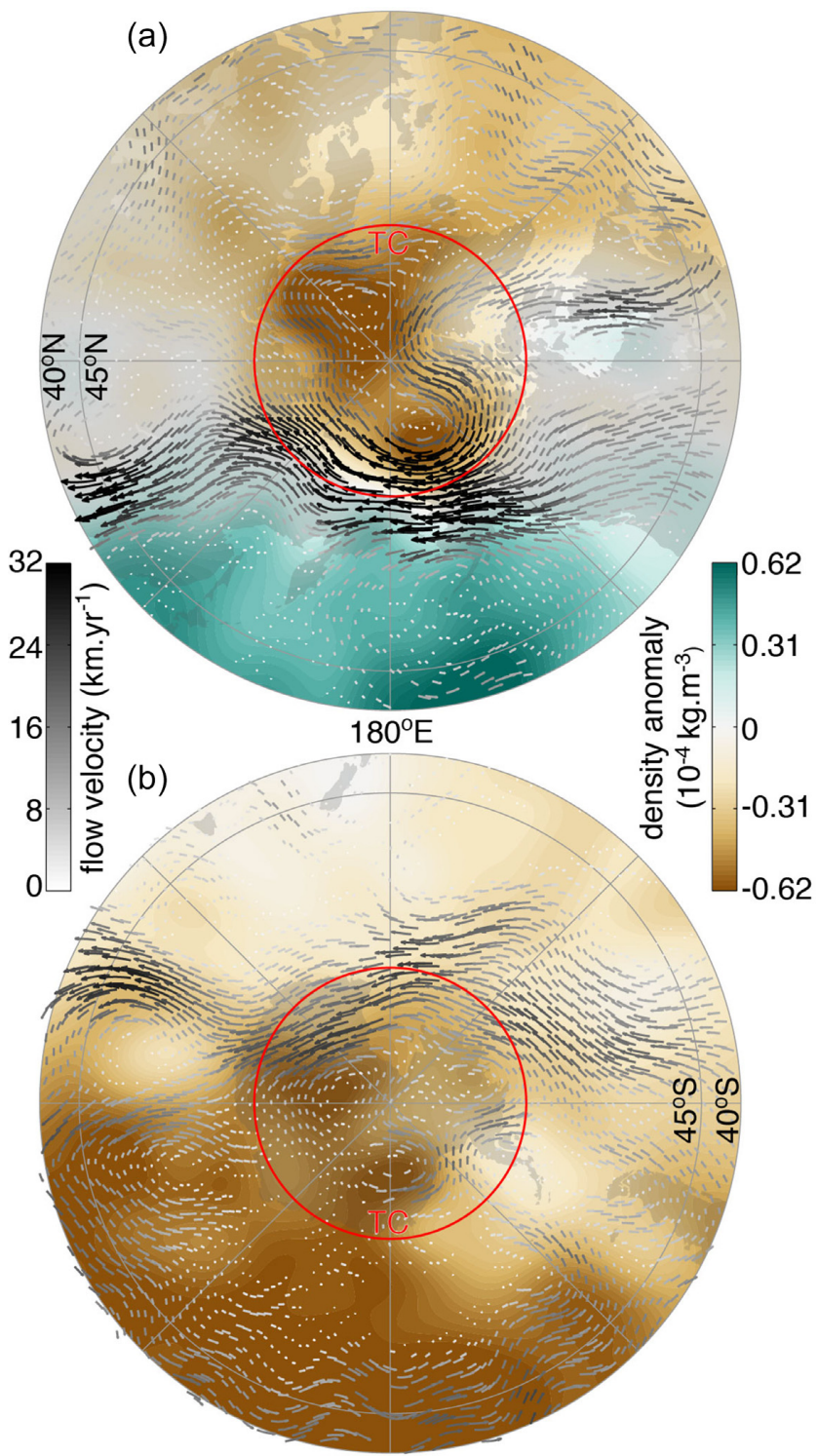

Figure 6. North (a) and south (b) polar projections of the ensemble averaged core surface flow obtained in 2015 in the mid-constraint inversion case (same conventions as in Fig. 4, overplotted on the ensemble averaged density anomaly pattern $C\left(r_{o}\right)$.

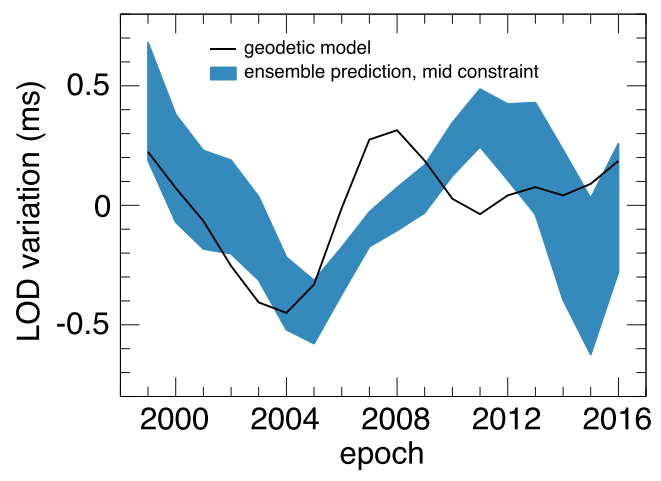

Figure 7. Core-originated variations of the length of day predicted by the ensemble of inverted core flows in the epoch range 1999-2016 (blue shaded \pm 1 std. dev. range), for the mid-constraint inversion case. See equations $(25,26)$ in Aubert et al. (2013) for the technical computation procedure, which only involves the radial profile $T_{1}^{0}(r)$. Also represented (black) is a geodetic model obtained from the IERS C04 timeseries (Bizouard \& Gambis 2009) after removal of contributions from atmospheric angular momentum, from solid tides, and from a $1.4 \mathrm{mscy}^{-1}$ secular linear trend (computation courtesy of N. Gillet). The model stops in 2016 because the reanalysis of external contributions is not available afterwards.
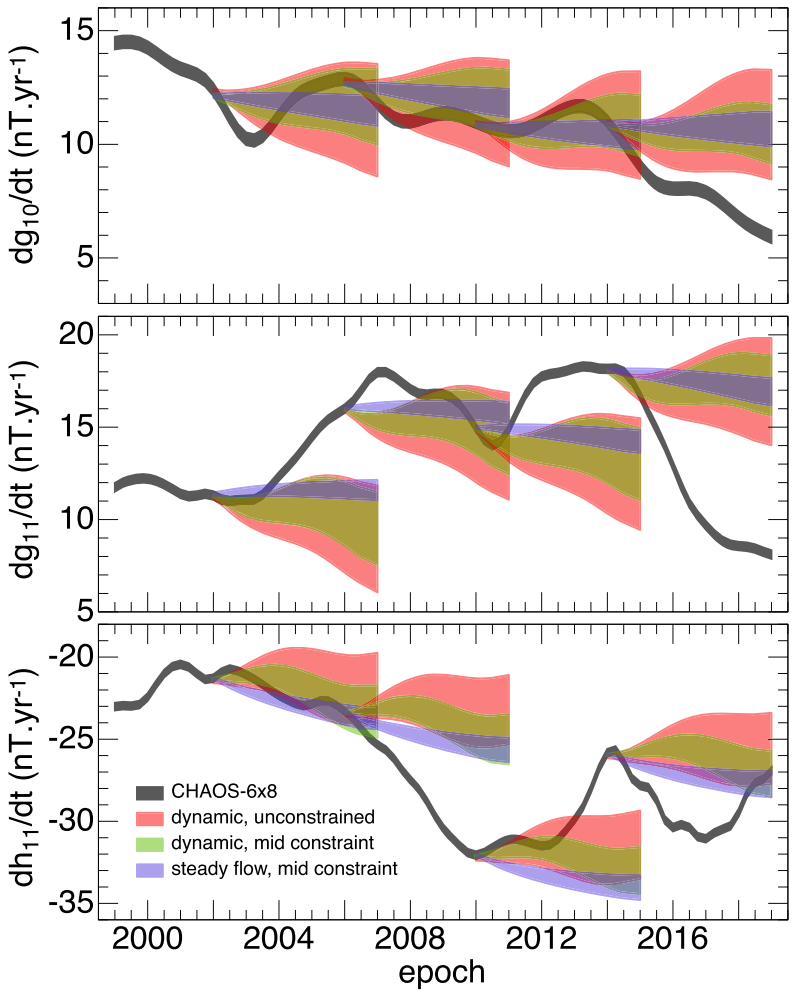

Figure 8. Temporal evolution of Gauss coefficients time derivatives $\partial_{t} g_{1}^{0}, \partial_{t} g_{1}^{1}, \partial_{t} h_{1}^{1}$ for the geomagnetic field model CHAOS-6x8 (grey shaded area representing the $\pm 1 S D$ of the error model described in Section 2.2), and for forecasts at 5-yr range obtained by numerically integrating the ensemble of core states obtained at various starting epochs (colour shaded regions representing the average \pm 1 std. dev. of ensemble forecasts). Shown are the results for three forecasting strategies: dynamic unconstrained (red), dynamic mid-constrained (green) and steady flow midconstrained (blue). 

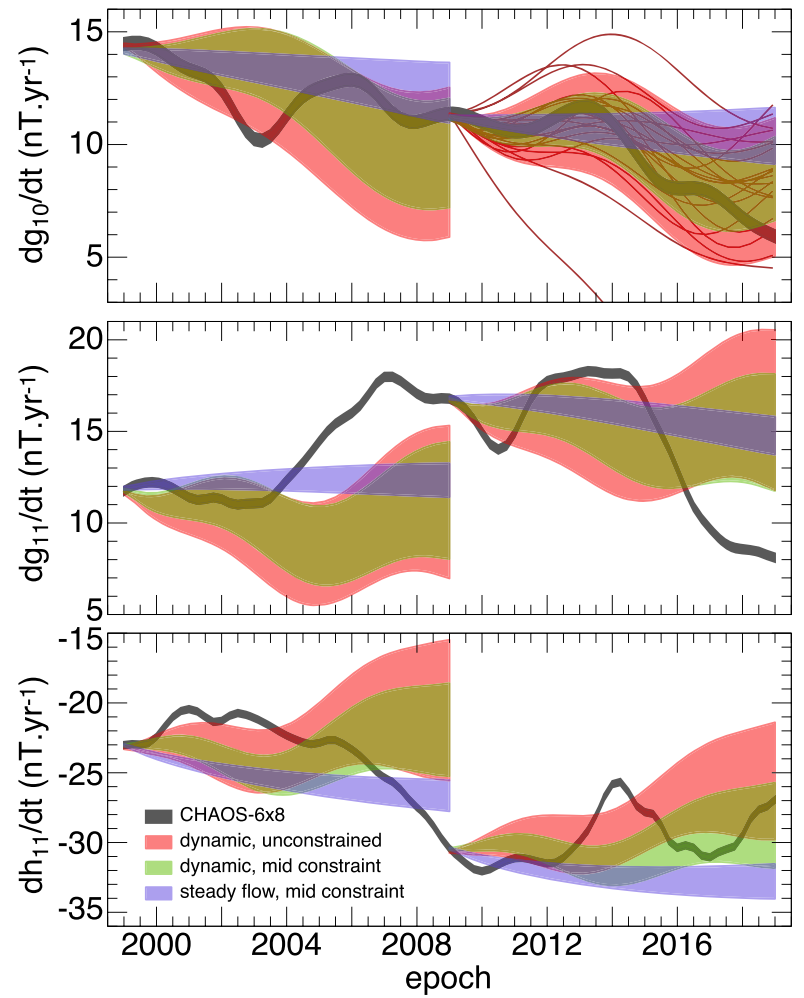

Figure 9. Same as Fig. 8, for forecasts at 10-yr range. Examples of individual ensemble trajectories are shown in the upper panel for the dynamic unconstrained forecast started in 2009.

of eq. (2):

$$
\frac{\partial^{2} \mathbf{B}}{\partial t^{2}}=\nabla \times\left(\frac{\partial \mathbf{u}}{\partial t} \times \mathbf{B}\right)+\nabla \times\left(\mathbf{u} \times \frac{\partial \mathbf{B}}{\partial t}\right)+\eta \nabla^{2}\left(\frac{\partial \mathbf{B}}{\partial t}\right) .
$$

The key to a successful forecast is to estimate a relevant geomagnetic acceleration $\partial_{t}^{2} \mathbf{B}$ by analysing data for the magnetic field $\mathbf{B}$, secular variation $\partial_{t} \mathbf{B}$ in order to correctly describe all terms on the righthand side of eq. (47). We recall that at subdecadal to decadal ranges, this acceleration is governed by several contributions (e.g. Aubert 2018). First, the slow convective evolution is governed by the QGMAC balance in eq. (1), with the time dependency being provided by the evolution of the magnetic field $\mathbf{B}$ and density anomaly field $C$ in eqs (2) and (3). In terms of contributions to the geomagnetic acceleration as broken down in eq. (47), this part arises from the contribution of the interaction between $\partial_{t} \mathbf{B}$ and $\mathbf{u}$ as well as that of the diffusive acceleration $\nabla^{2} \partial_{t} \mathbf{B}$. Secondly, deviations from the QG-MAC balance can intermittently lead to rapid accelerations driven by inertia in eq. (1), and by the interaction between flow acceleration $\partial_{t} \mathbf{u}$ and the magnetic field $\mathbf{B}$ in eq. (47). If the QG-MAC balance is adequately respected, then these deviations can have a geophysical significance, for instance to describe geomagnetic jerks resulting from magneto-inertial wave dynamics (Aubert \& Finlay 2019). However, if the balance is improperly rendered in the inverse states, then the resulting inertial and magneto-inertial dynamics is not physically relevant and deteriorates the forecast quality.

This last situation is indeed encountered in our forecasts where the QG-MAC force balance is initially not constrained, which reproduce behaviours previously reported in Aubert (2015). Examining the evolution of magnetic variation $\partial_{t} g_{1}^{0}, \partial_{t} g_{1}^{1}, \partial_{t} h_{1}^{1}$ for a set of large-scale Gauss coefficients (Figs 8 and 9) we indeed notice that the envelope of unconstrained ensemble forecasts tends to

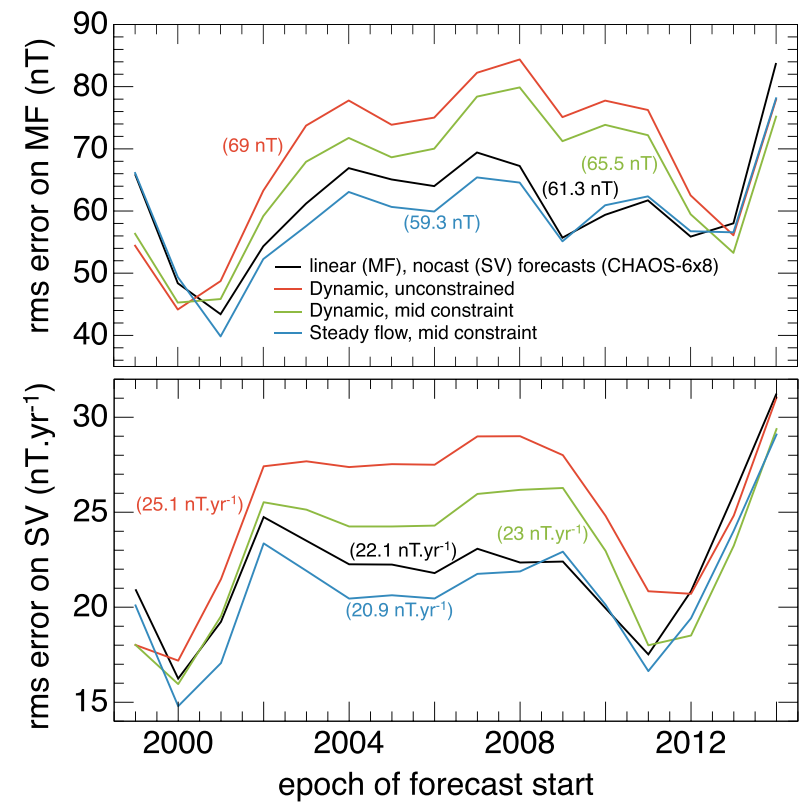

Figure 10. R.m.s difference at Earth surface between CHAOS-6x8 and the ensemble averaged magnetic field (MF, top) or secular variation (SV, bottom) obtained at the end of 5-yr forecasts, as a function of the epoch of forecast start. Represented are the results of dynamic unconstrained (red), dynamic mid-constrained (green) and steady flow (blue) forecasting strategies. Also reported are the results of a mathematical linear extrapolation of the MF from CHAOS-6x8 using its SV at the epoch of forecast start (black, top), and the results of a nocast prediction of the SV assuming zero acceleration (black, bottom). Numbers between parentheses indicate the level of error averaged over forecasts started each year in the epoch range 1999-2014.

oscillate at a natural period close to the planetary rotation period $2 \pi / \Omega=10.6$ yr of the coupled Earth model. This behaviour is best seen in the forecasts at 10-yr range (Fig. 9), where the examination of individual trajectories also reveals that the oscillation period is variable among ensemble members. These oscillations are the signature of uncontrolled inertial waves (Alfvén waves being absent at the start of path, Aubert 2018), and consistently with inertial wave theory (e.g. Greenspan 1968) we do not observe periods shorter than half that of planetary rotation. The spurious appearance of inertial waves is consistent with the value $\tau_{\text {flow }}=7.8 \mathrm{yr}$ obtained in unconstrained inversions (see Table 2). This is significantly shorter than the value $\tau_{\text {flow }}=24 \mathrm{yr}$ obtained in a free run of the coupled earth model (Aubert 2014), and also interferes with the range 5$10 \mathrm{yr}$ at which the geomagnetic evolution is predicted, which implies that the forecast quality is degraded by the presence of the inertial waves. In contrast, the amplitude of spurious inertial oscillations is reduced when imposing the QG-MAC constraint, consistently with the reduced levels of inertia obtained in Fig. 3(c) and with the larger value $\tau_{\text {flow }}=13.2 \mathrm{yr}$. Relative to the unconstrained situation, the r.m.s. forecast error at Earth's surface (Figs 10 and 11) is then markedly reduced in the constrained case. This last result confirms the relevance of the QG-MAC balance to describe Earth's core dynamics.

Figs 10 and 11 however show that the QG-MAC constrained dynamic forecasts still do not surpass the quality of naive mathematical predictions made by extrapolating the field using its rate of change at the start epoch (linear forecasts without magnetic acceleration), though the gap between the two is now rather narrow. To explain this it should be kept in mind that the constraint is only incompletely enforced at the core surface (Fig. 3c) and not at all in the volume. 


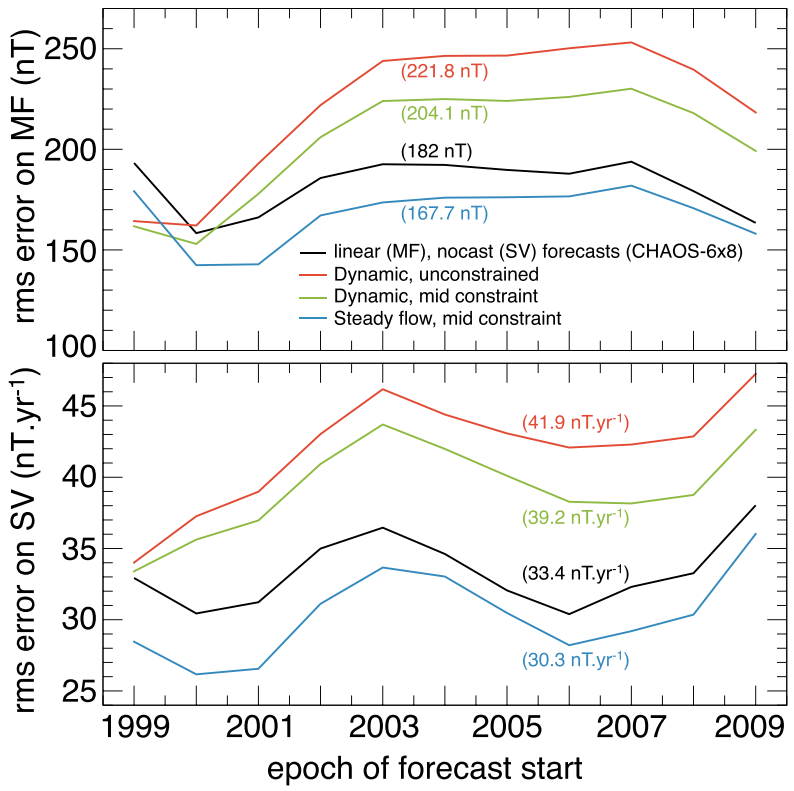

Figure 11. Same as Fig. 10, for forecasts at 10-yr range, with numbers between parentheses indicating the level of error averaged over the epoch range 1999-2009.

Introducing a steady flow forecast that is representative of the results that would be obtained if the QG-MAC balance was perfectly enforced throughout the core, we notice that the resulting predictions now slightly improve over the quality of the mathematical extrapolations. This shows that the estimation of the magnetic acceleration parts captured by a steady flow approach [i.e. those resulting from the interaction of $\mathbf{u}$ with $\partial_{t} \mathbf{B}$ and the diffusive acceleration $\nabla^{2} \partial_{t} \mathbf{B}$ in eq. (47)] is adding relevant information into the forecast. A closer inspection of the contributions to error from individual Gauss coefficients reveals that much of the improvement over mathematical extrapolations is carried by the large-scale coefficients represented in Figs 8 and 9, where the steady flow forecasts frequently capture the correct future trend, though with a lower acceleration than that required by the data. The missing part underlines the importance of the geomagnetic acceleration resulting from the interaction of $\partial_{t} \mathbf{u}$ with $\mathbf{B}$ in eq. (47), which can only be correctly rendered if levels of residual inertia are reduced enough relative to the MAC forces in order to enable the correct magnetohydrodynamic wave dynamics at interannual timescales (Aubert 2018; Aubert \& Finlay 2019). This goal is outside the scope of the dynamics rendered by the coupled earth model because of its position on the parameter space path.

\section{DISCUSSION}

\subsection{Force balance in Earth's core}

In this study, an inverse geodynamo framework has been extended in order to quantitatively assess the compatibility of recent geomagnetic main field and secular variation data with the prior hypothesis of a QG-MAC force balance in Earth's core. This hypothesis is to some extent already embedded into the statistics used to constrain the inversion, since these are obtained from a numerical dynamo model that already respects this balance. We have seen indeed (Fig. 3) that the sole specification of statistics already leads to states that respect the linear part of the QG-MAC balance, that is the thermal wind equilibrium between the pressure gradient, buoyancy and
Coriolis forces. However, the Bayesian inference that is used in the framework is not sufficient to account for the nonlinear effects of the Lorentz force. Enforcing the full QG-MAC balance through a direct implementation in the hydrodynamic inverse problem, with nonlinear Lorentz terms being explicitly treated (eq. 26), we have seen that it is possible to obtain core states that adequately fit the geomagnetic data (Figs 1 and 2, Table 2) while producing residuals of the QG-MAC balance smaller than each term in this balance (Fig. 3). As these residuals induce a spurious inertial response, decreasing their amplitude is also systematically beneficial when performing forecasts of the future geomagnetic evolution by using the inverted states as startup points (Figs 8-11). Up to spherical harmonic degree $\ell_{\mathrm{asm}}=30$, and at the core surface, the Lorentz force is found to be subdominant relative to the pressure, Coriolis and buoyancy forces (Fig. 3), thereby confirming the absence of system-scale magnetostrophy (equilibrium between the magnetic and pressure force). All these elements complement the bulk of recent direct modelling results (Aubert et al. 2017; Schwaiger et al. 2019) and provide further data-driven support to the proposal of a QG-MAC force balance controlling the geodynamo.

\subsection{General circulation in Earth's core and its main driving source}

The core flow solutions constrained with the QG-MAC balance confirm the general circulation features found in previous studies (Pais \& Jault 2008; Gillet et al. 2013, 2015, 2019; Aubert 2013, 2014, 2015; Pais et al. 2015; Barrois et al. 2017; Livermore et al. 2017; Bärenzung et al. 2018), and again highlight a planetaryscale, eccentric columnar gyre in Earth's core, the surface signature of which consists of a strong equatorial westward drift in the Atlantic hemisphere and an intense, roughly equator-symmetric jet at high latitudes in the Pacific hemisphere (Fig. 4). By directly imposing dynamical consistency into the inverse problem, the present results confirm that this circulation is driven by buoyancy, rather than magnetic forces (Fig. 3). At the core surface, the longitudinal hemispheric flow circulation is therefore in thermal wind balance with a similarly hemispherical density anomaly distribution (Fig. 6). Similarly to Gillet et al. (2019), our results also incite to revise the amount of acceleration seen in the high-latitude Pacific jet during the past two decades. During this period indeed (Fig. 5), we find significant acceleration only in the Northern Hemisphere, with an average value of $0.4 \mathrm{~km} \mathrm{yr}^{-2}$ that is more than four times smaller than that reported by Livermore et al. (2017). This is probably the consequence of our inversions accounting for truncation errors and magnetic diffusion effects. While the strong accelerations found by Livermore et al. (2017) were supportive of a magnetic driving of the flow, we note that with a typical jet velocity of $30 \mathrm{~km} \mathrm{yr}^{-1}$, the acceleration timescale of the gyre is at least $30 / 0.4=75 \mathrm{yr}$, a value compatible with convective advection of density anomalies.

The geophysical origin of the striking hemispherical buoyancy pattern (Fig. 6) is not yet entirely clear. As in our earlier work (Aubert 2013; 2014; Aubert et al. 2013), the present results confirm that the hypothesis of generating this pattern through faster inner core freezing in the Eastern Hemisphere $\left(0^{\circ} \mathrm{E}-180^{\circ} \mathrm{E}\right)$ is reasonably compatible with the geomagnetic data. The geodynamic justification for differential inner core freezing initially came from the idea that the inner core surface should be considered as an open surface permeable to transfers of matter (Monnereau et al. 2010), and that the solid inner core could then be subject to translational convective instabilities. This however requires the background density pattern 
of the inner core to be convectively unstable, a possibility which has been questioned owing to the ongoing debate on the value of thermal conductivity in the inner and outer core (e.g. Pozzo et al. 2012; Konôpková et al. 2016). In the event that the inner core is thermally stratified, it has been recently underlined that it can still be chemically unstably stratified because of a time-dependent light element partitioning between the solid and the liquid as the inner core grows (Gubbins et al. 2013). In this case, the net stratification can be unstable and the resulting translational hemispherical anomalies in the inner core freezing rate may be of the same order as the homogeneous freezing rate (Deguen et al. 2018). This is precisely the situation explored in the coupled Earth model, and the present inverse geodynamo modelling results then provide a consistent buoyancy-driven explanation of the eccentric gyre and highlatitude jet. As we have seen in the cases when we strongly enforce the QG-MAC dynamic constraint in our inversions, this explanation however still leaves room for improvement. In this situation indeed, the coupled earth model typically favours strong upwellings in the Eastern Hemisphere, which are not entirely consistent with the recent secular variation data (Fig. 2). Furthermore, the possibility still remains that the hemispherical circulation spontaneously arises in a homogeneously forced system, as shown by the recent simulations of Schaeffer et al. (2017) at extreme conditions. The tool presented here opens interesting prospects towards further investigation of this issue, that could for instance be explored by changing the prior model underlying the inversions in order to assess the compatibility of each scenario with the available data. It is also important to promote the acquisition of additional archeomagnetic information on the historical geomagnetic field in order to assess the persistence of the eccentric gyre over several centuries.

\subsection{Towards predictions of geomagnetic jerks}

We have seen that enforcing the QG-MAC balance is an essential prerequisite to accurately render the geomagnetic field evolution at subdecadal to decadal timescales. Once the inertial residuals of this balance have been sufficiently decreased indeed, the information brought by the numerical dynamo prior leads to relevant partial estimates of the geomagnetic acceleration and to forecasts that can slightly improve over the mathematical linear extrapolations (Figs 8-11). While the present approach therefore represents a step towards better geomagnetic forecasts, it remains limited as we have only achieved a moderate reduction of the inertial residuals compared to the unconstrained situation (Fig. 3). In the present scheme, the QG-MAC balance is also enforced only at the core surface. Another problem is that the estimation of the internal magnetic field and the associated Lorentz force remains of statistical nature, and its compatibility with the other force components (buoyancy, Coriolis) is not guaranteed, leading to an increase of the misfits in the constrained situation (Table 2). Co-estimation of the magnetic field, flow and buoyancy anomaly, possibly in an iterative scheme, represents a viable avenue for further developing the inverse geodynamo modelling framework.

With inertial forces at least five orders of magnitude below the MAC forces (Aubert et al. 2017; Aubert 2019) in Earth's core, achieving a realistic level of the QG-MAC balance and relevant geomagnetic forecasts including Alfvén-wave driven dynamics is a very delicate task. It is however essential to reach this level of accuracy if one wishes to be able to predict geomagnetic jerks, which may result from tiny magneto-inertial deviations to the QGMAC balance that propagate as quasi-geostrophic Alfvén waves and undergo an amplification effect as they reach the core surface (Aubert \& Finlay 2019). In that sense, future progress towards this fundamental goal of geomagnetism should come from parallel improvements in the three pillars of geomagnetic prediction: additional satellite geomagnetic data providing insight into a larger number of rapid geomagnetic acceleration events, better direct numerical models that operate at the correct level of inertial forces, and refinements in data assimilation frameworks able to infer core states with a finer level of dynamic balance.

\section{ACKNOWLEDGEMENTS}

J.A. wishes to thank two anonymous referees for comments that helped to improve the manuscript, and Nicolas Gillet for discussions and for providing the geodetic model for core-originated variations in the length of day. Support is acknowledged from the Fondation Simone et Cino Del Duca of Institut de France (2017 Research Grant) and from the European Space Agency through contract 4000127193/19/NL/IA. Numerical computations were performed at S-CAPAD, IPGP and using HPC resources from GENCI-TGCC and GENCI-CINES (Grant numbers A0040402122 and A0060402122). The results presented in this work rely on data collected by satellites and at magnetic observatories. J.A. thanks the national institutes that support them and INTERMAGNET for promoting high standards of magnetic observatory practice (www.intermagnet.org).

\section{REFEREN CES}

Amit, H. \& Pais, M.A., 2013. Differences between tangential geostrophy and columnar flow, Geophys. J. Int., 194(1), 145-157.

Aubert, J., 2013. Flow throughout the Earth's core inverted from geomagnetic observations and numerical dynamo models, Geophys. J. Int., 192(2), 537-556.

Aubert, J., 2014. Earth's core internal dynamics 1840-2010 imaged by inverse geodynamo modelling, Geophys. J. Int., 197(3), 1321-1334.

Aubert, J., 2015. Geomagnetic forecasts driven by thermal wind dynamics in the Earth's core, Geophys. J. Int., 203(3), 1738-1751.

Aubert, J., 2018. Geomagnetic acceleration and rapid hydromagnetic wave dynamics in advanced numerical simulations of the geodynamo, Geophys. J. Int., 214(1), 531-547.

Aubert, J., 2019. Approaching Earth's core conditions in high-resolution geodynamo simulations, Geophys. J. Int., 219(S1), S137-S151.

Aubert, J. \& Finlay, C.C., 2019. Geomagnetic jerks and rapid hydromagnetic waves focusing at Earth's core surface, Nat. Geosci., 12(5), 393-398.

Aubert, J., Finlay, C.C. \& Fournier, A., 2013. Bottom-up control of geomagnetic secular variation by the Earth's inner core, Nature, 502, 219-223.

Aubert, J., Gastine, T. \& Fournier, A., 2017. Spherical convective dynamos in the rapidly rotating asymptotic regime, J. Fluid. Mech., 813, 558-593.

Aurnou, J.M. \& King, E.M., 2017. The cross-over to magnetostrophic convection in planetary dynamo systems, Proc. Roy. Soc. A, 473(2199), doi:10.1098/rspa.2016.0731.

Bärenzung, J., Holschneider, M., Wicht, J., Sanchez, S. \& Lesur, V., 2018. Modeling and predicting the short-term evolution of the geomagnetic field, J. geophys. Res., 123(6), 4539-4560.

Barrois, O., Gillet, N. \& Aubert, J., 2017. Contributions to the geomagnetic secular variation from a reanalysis of core surface dynamics, Geophys. J. Int., 211(1), 50-68.

Beggan, C. \& Whaler, K., 2010. Forecasting secular variation using core flows, Earth, Planets Space, 62(10), 11.

Bizouard, C. \& Gambis, D., 2009. The Combined Solution C04 for Earth Orientation Parameters Consistent with International Terrestrial Reference Frame 2005, pp. 265-270, Springer Berlin Heidelberg.

Calkins, M.A., 2018. Quasi-geostrophic dynamo theory, Phys. Earth. Planet. Int., 276, 182-189. 
Calkins, M.A., Julien, K., Tobias, S.M. \& Aurnou, J.M., 2015. A multiscale dynamo model driven by quasi-geostrophic convection, J. Fluid. Mech., 780, 143-166.

Christensen, U.R., Aubert, J. \& Hulot, G., 2010. Conditions for Earth-like geodynamo models, Earth. planet. Sci. Lett., 296(3-4), 487-496.

Christensen, U.R., Wardinski, I. \& Lesur, V., 2012. Timescales of geomagnetic secular acceleration in satellite field models and geodynamo models, Geophys. J. Int., 190(1), 243-254.

Davidson, P.A., 2013. Scaling laws for planetary dynamos, Geophys. J. Int., 195(1), 67-74.

Deguen, R., Alboussière, T. \& Labrosse, S., 2018. Double-diffusive translation of Earth's inner core, Geophys. J. Int., 214(1), 88-107.

Dormy, E., 1997, Modélisation numérique de la dynamo terrestre, $P h D$ thesis, Institut de Physique du Globe de Paris, http://www.math.ens.fr $/ \sim \mathrm{d}$ ormy/Publications/doubleTh.html, see also Errata.

Dormy, E., 2016. Strong-field spherical dynamos, J. Fluid. Mech., 789, 500-513.

Evensen, G., 2003. The ensemble Kalman filter: theoretical formulation and practical implementation, Ocean Dyn., 53, 343-367.

Eymin, C. \& Hulot, G., 2005. On core surface flows inferred from satellite magnetic data, Phys. Earth planet. Int., 152, 200-220.

Finlay, C.C., Aubert, J. \& Gillet, N., 2016a. Gyre-driven decay of the Earth's magnetic dipole, Nat. Comm., 7, doi:10.1038/ncomms10422.

Finlay, C.C., Olsen, N., Kotsiaros, S., Gillet, N. \& Tøffner-Clausen, L., 2016b. Recent geomagnetic secular variation from Swarm and ground observatories as estimated in the CHAOS-6 geomagnetic field model, Earth, Planets Space, 68(1), 112.

Finlay, C.C., Jackson, A., Gillet, N. \& Olsen, N., 2012. Core surface magnetic field evolution 2000-2010, Geophys. J. Int., 189(2), 761-781.

Fournier, A., Aubert, J. \& Thébault, E., 2015. A candidate secular variation model for IGRF-12 based on Swarm data and inverse geodynamo modelling, Earth. Planets. Space., 67, 81.

Fournier, A., Aubert, J. \& Thébault, E., 2011. Inference on core surface flow from observations and 3-D dynamo modelling, Geophys. J. Int., 186(1), $118-136$.

Gillet, N., Jault, D. \& Finlay, C.C., 2015. Planetary gyre, time-dependent eddies, torsional waves and equatorial jets at the Earth's core surface, $J$. geophys. Res., 120, 3991-4013.

Gillet, N., Huder, L. \& Aubert, J., 2019. A reduced stochastic model of core surface dynamics based on geodynamo simulations, Geophys. J. Int., 219(1), 522-539.

Gillet, N., Pais, M.A. \& Jault, D., 2009. Ensemble inversion of timedependent core flow models, Geochem. Geophys. Geosyst., 10 (6), doi:10.1029/2008GC002290.

Gillet, N., Jault, D., Canet, E. \& Fournier, A., 2010. Fast torsional waves and strong magnetic field within the Earth's core, Nature, 465(7294), 74-77.

Gillet, N., Jault, D., Finlay, C.C. \& Olsen, N., 2013. Stochastic modeling of the Earth's magnetic field: Inversion for covariances over the observatory era, Geochem. Geophys. Geosyst., 14(4), 766-786.

Greenspan, H.P., 1968. The Theory of Rotating Fluids, Breukelen Press.

Gubbins, D., Alfe, D. \& Davies, C.J., 2013. Compositional instability of Earth's solid inner core, Geophys. Res. Lett., 40(6), 1084-1088.

Hollerbach, R., 1996. On the theory of the geodynamo, Phys. Earth Planet. Int., 98(3-4), 163-185.

Holme, R. \& Olsen, N., 2006. Core surface flow modelling from highresolution secular variation, Geophys. J. Int., 166(2), 518-528.
Hulot, G., Eymin, C., Langlais, B., Mandea, M. \& Olsen, N., 2002. Smallscale structure of the geodynamo inferred from Oersted and Magsat satellite data, Nature, 416, 620-623.

Hulot, G., Sabaka, T.J., Olsen, N. \& Fournier, A., 2015. The present and future geomagnetic field, in Treatise on Geophysics, 2nd edn, Vol. 5, pp. 33-78, Elsevier.

Jackson, A., 1997. Time-dependency of tangentially geostrophic core surface motions, Phys. Earth planet. Int., 103(3-4), 293-311.

Kloss, C. \& Finlay, C.C., 2019. Time-dependent low-latitude core flow and geomagnetic field acceleration pulses, Geoph. J. Int., 217(1), 140-168.

Konôpková, Z., McWilliams, R.S., Gómez-Pérez, N. \& Goncharov, A.F., 2016. Direct measurement of thermal conductivity in solid iron at planetary core conditions, Nature, 534(7605), 99-101.

Le Mouël, J.-L., 1984. Outer-core geostrophic flow and secular variation of earths geomagnetic-field, Nature, 311(5988), 734-735.

Le Mouël, J.-L., Gire, C. \& Madden, T., 1985. Motions at core surface in the geostrophic approximation, Phys. Earth planet. Int., 39(4), 270-287.

Lesur, V., Wardinski, I., Baerenzung, J. \& Holschneider, M., 2017. On the frequency spectra of the core magnetic field gauss coefficients, Phys. Earth Planet. Int., 276, 145-158.

Lhuillier, F., Fournier, A., Hulot, G. \& Aubert, J., 2011. The geomagnetic secular-variation timescale in observations and numerical dynamo models, Geophys. Res. Lett., 38, L09306.

Livermore, P.W., Hollerbach, R. \& Finlay, C.C., 2017. An accelerating highlatitude jet in Earth's core, Nat. Geosci., 10(1), 62-68.

Monnereau, M., Calvet, M., Margerin, L. \& Souriau, A., 2010. Lopsided growth of Earth's inner core, Science, 328(5981), 1014-1017.

Pais, M.A. \& Jault, D., 2008. Quasi-geostrophic flows responsible for the secular variation of the Earth's magnetic field, Geophys. J. Int., 173(2), 421-443.

Pais, M.A., Oliveira, O. \& Nogueira, F., 2004. Nonuniqueness of inverted core-mantle boundary flows and deviations from tangential geostrophy, J. geophys. Res., 109(B8), doi:10.1029/2004JB003012.

Pais, M.A., Morozova, A.L. \& Schaeffer, N., 2015. Variability modes in core flows inverted from geomagnetic field models, Geophys. J. Int., 200(1), 402-420.

Pozzo, M., Davies, C.J., Gubbins, D. \& Alfè, D., 2012. Thermal and electrical conductivity of iron at Earth's core conditions, Nature, 485(7398), 355358.

Sabaka, T.J., Olsen, N., Tyler, R.H. \& Kuvshinov, A., 2015. CM5, a preswarm comprehensive geomagnetic field model derived from over $12 \mathrm{yr}$ of CHAMP, œrsted, SAC-C and observatory data, Geophys. J. Int., 200, 1596-1626.

Schaeffer, N., 2013. Efficient spherical harmonic transforms aimed at pseudospectral numerical simulations, Geophys. Geochem. Geosyst., 14(3), 751-758.

Schaeffer, N., Jault, D., Nataf, H.-C. \& Fournier, A., 2017. Turbulent geodynamo simulations: a leap towards Earth's core, Geophys. J. Int., 211(1), $1-29$.

Schwaiger, T., Gastine, T. \& Aubert, J., 2019. Force balance in numerical geodynamo simulations: a systematic study, Geophys. J. Int., 219(S1), S101-S114.

Wardinski, I. \& Lesur, V., 2012. An extended version of the C3FM geomagnetic field model: application of a continuous frozen-flux constraint, Geophys. J. Int., 189(3), 1409-1429.

Whaler, K.A. \& Beggan, C.D., 2015. Derivation and use of core surface flows for forecasting secular variation, J. geophys. Res., 120(3), 1400-1414. 